\title{
Mathematical Model for Spatial Segregation of the Rho-Family GTPases Based on Inhibitory Crosstalk
}

\author{
Alexandra Jilkine $^{\mathrm{a}, *}$, Athanasius F.M. Marée ${ }^{\mathrm{b}}$, Leah Edelstein-Keshet ${ }^{\mathrm{a}}$ \\ ${ }^{a}$ Department of Mathematics and Institute of Applied Mathematics, University of British \\ Columbia, Vancouver, BC V6T 1Z2, Canada \\ ${ }^{b}$ Theoretical Biology/Bioinformatics, Utrecht University, Utrecht, The Netherlands
}

Received: 5 June 2006 / Accepted: 12 January 2007

(C) Society for Mathematical Biology 2007

\begin{abstract}
Cdc42, Rac, and Rho are small GTPases known to play a central role in signal transduction to the actin cytoskeleton. These proteins regulate cell motility, by affecting nucleation, uncapping, and depolymerization of actin filaments, and acto-myosin contractility. Studies of crosstalk and mutual feedbacks in these three proteins have led to a number of proposals for their interaction. At the same time, observations of the spatiotemporal dynamics of Rho-family proteins give evidence of spatial polarization and mutual exclusion between Cdc42/Rac and Rho. In this paper, we formulate a mathematical model to account for such observations, based on the known underlying biology of these proteins. We first investigate which of the crosstalk schemes proposed in the literature is consistent with observed dynamics, and then derive a simple model that can correctly describe these dynamics (assuming crosstalk is mediated via Rho GEFs). We show that cooperativity is an essential ingredient in the interactions of the proteins. The co-occurrence of a stable rest state with the possibility of fast spatial segregation can be related to bistability in a set of underlying ODEs in which the inactive forms of these proteins are fixed at a constant level. We show that the fast diffusion of the inactive forms is essential for stabilizing the transition fronts in the PDE formulation of the model, leading to robust spatial polarization, rather than traveling waves.
\end{abstract}

Keywords Cdc42 · Rac · Rho · Rho-family GTPases · Cell polarization · Molecular switch $\cdot$ Spatiotemporal distribution $\cdot$ Mathematical model

\section{Introduction}

The ability to move is a fundamental attribute of both prokaryotic and eukaryotic cells. Cell motility is vital for fetal development, wound repair, and functioning of the immune system. During cell migration, the cell must interpret extracellular cues to elicit complex

\footnotetext{
*Corresponding author.

E-mail address: jilkine@math.ubc.ca (Alexandra Jilkine).
} 
intracellular changes, and to establish a polarity with a distinguishable front and back. Recently, small GTPases (G-proteins) of the Rho family have emerged as key intracellular players in regulating cell polarity. In a separate treatment (Dawes and Edelstein-Keshet, 2007) we have explored their interactions with another family of polarity regulators, the phosphoinositides. The three best-studied members of the Rho family are Cdc42, Rac1 and RhoA, which are ubiquitously expressed and assumed to be essential players in cytoskeletal organization in higher organisms, although divergent findings are also current in the literature. ${ }^{1}$ These proteins cycle between an active GTP-bound state on the membrane, and an inactive GDP-bound state on the membrane or in the cytosol, acting as molecular switches. Figure 1 depicts the basic biochemistry. Guanine nucleotide exchange factors (GEFs) activate small GTPases by promoting replacement of GDP by GTP, while GTPase activating proteins (GAPs) accelerate GTP hydrolysis and inactivate the proteins (Raftopoulou and Hall, 2004).

One of the driving forces behind cell motility is the polymerization and depolymerization of the actin cytoskeleton, a highly dynamic network of protein filaments found in all eukaryotic cells (Mitchison and Cramer, 1996). In their active forms, Rho GTPases regulate the activity of numerous proteins that affect the cytoskeleton, forming a link to the assembly and dynamics of filamentous actin (Mackay and Hall, 1998). Active Rac stimulates branching and polymerization of actin filaments by the Arp $2 / 3$ complex that is known to initiate branch points on the sides of pre-existing actin filaments (Miki and Takenawa, 2003). Rac inhibits capping of actin filament barbed ends close to the plasma membrane via the phosphoinositide $\mathrm{PIP}_{2}$ (Tolias et al., 2000). Rac also stimulates LIM-kinase that inactivates the filament degrading protein, cofilin (Stanyon and Bernard, 1999). Like Rac, Cdc42 stimulates actin polymerization by a pathway leading to activation of the Arp2/3 complex (Rohatgi et al., 1999). Rho stimulates myosin light chain (MLC) phosphorylation via its downstream target Rho kinase (ROCK) (Kimura et al., 1996). The increased level of phosphorylated MLC promotes the interaction of myosin filaments with actin filaments and increases contractility. The signal-transduction pathways from the Rho proteins to the cytoskeleton are further reviewed in Bishop and Hall (2000), Matozaki et al. (2000), Ridley (2001a, 2001b).

Fluorescence data suggests that different Rho proteins are spatially segregated in a polarized cell. Kraynov et al. (2000) reported that in migrating Swiss 3T3 fibroblasts, active Rac forms a gradient and is highest at the leading edge of the cells. Similar experiments revealed a high level of active Cdc42 at cell edges that are undergoing remodeling (Nalbant et al., 2004). Furthermore, there is a close correlation between extension and retraction of individual protrusions and the rise and fall of Cdc42 activation. Itoh et al. (2002) detected gradients of active Cdc 42 and Rac that were highest at the leading edge in motile HT1080 cells. In contrast, Rho levels are highest at the back of the cell (Xu et al., 2003). Sharp spatial segregation between Cdc42 and Rho activity zones is also observed in Xenopus oocytes (Benink and Bement, 2005). It is generally believed that the distribution of active Rho is inverse to that of Cdc42 and Rac in motile cells (Raftopoulou and Hall, 2004). In neutrophils devoid of Cdc42/Rac activator (GEF) $\alpha$ Pix, active Rho is found all over the

\footnotetext{
${ }^{1}$ Recent experimental data indicates that additional (currently unknown) Rho family GTPases are involved in the establishment and maintenance of cell polarity during directed migration (Czuchra et al., 2005; Vidali et al., 2006; Wheeler et al., 2006). Hence, all further discussion refers to subfamilies of Cdc42, Rac, and Rho that share common GEFs.
} 
cell, not just at the back, implying that Cdc42/Rac activity downregulates Rho activity at the front (Li et al., 2005).

Classic experiments in fibroblasts have shown that there is a cascade of small G-protein activation, where active Cdc42 activates Rac, which activates Rho, although biochemical mechanisms for this cascade remained unknown (Nobes and Hall, 1995). A recent study shows that one mechanism through which activation of Cdc42 leads to activation of Rac, is allosteric regulation of a Rac-GEF Cool-2 (Baird et al., 2005). (Another possible mechanism is discussed in DerMardirossian et al., 2004.) After an initial report of Rac activating Rho in fibroblasts, many groups reported antagonism between Rac and Rho (Van Leeuwen et al., 1997; Sander et al., 1999; Evers et al., 2000; Zondag et al., 2000; Li et al., 2002; Caron, 2003; Meili and Firtel, 2003). ${ }^{2}$ Rac has also been reported to be essential for Rho activation in neutrophils (Pestonjamasp et al., 2006). Cdc42 has been found to both positively and negatively regulate Rho activation (Sander et al., 1999; Benink and Bement, 2005). Rho has also been found to be a local antagonist to Cdc42 (Benink and Bement, 2005). Figure 3 summarizes the various schemes for Rho protein interactions proposed in the literature.

One of the goals of this paper is to determine which of the many interaction schemes proposed in the literature are consistent with observed dynamics of the proteins. We will show that some of these schemes are incomplete, or have incorrect elements, whereas others are able to account for the observed spatial segregation of the GTPases. To do so, we assume that crosstalk between the small GTPases involves effects from active forms of these proteins onto the GEFs that mediate the activation of the others, but in Appendices $\mathrm{A}-\mathrm{C}$ we show that the same conclusions can be drawn when the interactions take place through the GAPs. We pick the simplest scheme that has the appropriate feedback elements. In that scheme, mutual inhibition of $\mathrm{Cdc} 42 / \mathrm{Rac}$ and Rho leads to a bistable system when the levels of the inactive forms are fixed. We investigate under what circumstances this could give rise to spatial polarization, and develop a model that explains the segregation of Rho GTPases observed in motile cells (such as neutrophils), consistent with the known biology of the Rho proteins. We avoid invoking unknown hypothetical molecules in our modeling.

We will show that the initiation of polarity can, indeed, stem from crosstalk interactions between Cdc42, Rac and Rho. However, were it not for the rapid cytosolic diffusion of the inactive (GDI-bound) forms of the small G-proteins, that polarity would not be maintained. Due to the rapid spatial communication stemming from those inactive forms, the transition zones that would ordinarily sweep across the domain are stabilized. Our model explains these phenomena and makes experimentally testable predictions.

\section{Model formulation for a single Rho protein}

\subsection{Basic kinetics in the absence of crosstalk}

Rho GTPases cycle between GDP-bound (inactive) and GTP-bound (active) conformations, and between cytosolic and membrane-bound forms. These cycles are regulated

\footnotetext{
${ }^{2}$ Caron (2003) reported that activation of Rac results in a three to four-fold decrease in the levels of active GTP-bound Rho, Zondag et al. (2000) found that downregulation of Rac increases Rho activity, and Meili and Firtel (2003) found that Rac and Rho mutually inhibit each other.
} 


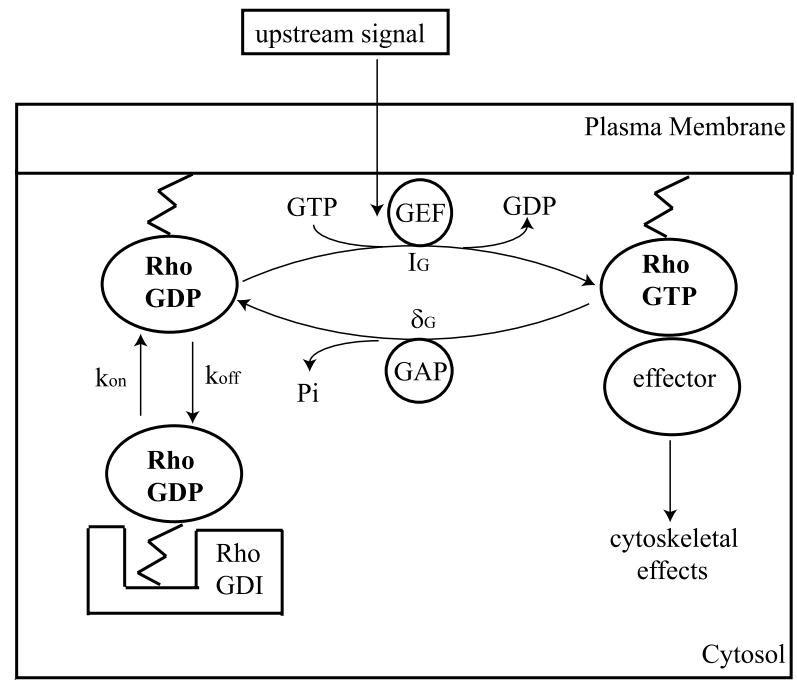

Fig. 1 A schematic diagram of the activation and membrane translocation cycles of a typical Rho family GTPase. In accordance with current literature, only the inactive form, Rho-GDP, can come off and on the membrane. To move into the cytosol, Rho-GDP needs to bind to a GDI molecule. Nucleotide exchange can only take place on the membrane. Dissociation of GDP from the GDP-bound form is an extremely slow reaction, so for Rho GTPases to be effective molecular switches, GDP dissociation is stimulated by various GEFs. Although the GDP/GTP exchange reaction is reversible, in reality, it almost never occurs. Instead, GTP is hydrolyzed to GDP, a process that is stimulated by various GAPs. Only the active, GTP-bound, forms of the proteins can bind to their effectors to have downstream effects or crosstalk with other GTPases.

by GEFs (guanine nucleotide exchange factors), GAPs (GTPase-activating proteins) and GDIs (GDP dissociation inhibitors). The dissociation of GDP from the GDP-bound form is an extremely slow reaction, greatly accelerated by GEFs. GEFs are typically recruited to stimulated receptors, where they become active, and, in turn, activate the Rho proteins. GAPs stimulate the rate of GTP hydrolysis of the Rho proteins, causing their inactivation. The G-protein has to be both in the GTP-configuration and membrane bound to interact with its effectors and trigger a cellular response (Takai et al., 2001). The third class of regulatory proteins, GDIs, inhibit the dissociation of the guanine nucleotide, keeping the proteins in one conformation. GDIs also extract the GDP-bound form of Rho proteins from the membrane to the cytosol, where they are sequestered in an inactive pool. The currently accepted biochemical model is that the GDP-bound form of the Rho proteins is present in the cytosol in an inactive 1:1 complex with GDI (Olofsson, 1999). The regulation of Rho GTPase activity is summarized in Fig. 1.

We make the following biologically reasonable assumptions:

1. The Rho proteins are not synthesized or degraded during cell polarization, but only undergo conversion between active and inactive forms. Therefore, the total amount of protein remains constant over the time scale on which the cell polarizes. This assumption is reasonable, because keratocyte cell fragments lacking a nucleus are still perfectly able to polarize rapidly and to move in response to a mechanical stimulus (Verkhovsky et al., 1999). 
2. The membrane diffusion coefficients of $\mathrm{Cdc} 42$, Rac, and Rho are roughly equal, because the three proteins are of a similar size and structure (Zerial and Huber, 1995).

3. Cytosolic forms diffuse approximately 100 times faster than the membrane-bound forms (Postma et al., 2004). As a result, the distribution of the cytosolic forms will be approximately homogeneous in space, even when the active forms are polarized on the membrane.

4. The amount of GDI present in the cell is sufficiently large that it is not be a limiting factor. Furthermore, switching between membrane and cytosolic states is very rapid (Sako et al., 2000).

5. Each Rho protein has a basal rate of activation (GTP-loading), and a basal turnover or inactivation rate (GTP-hydrolysis). Activation and inactivation of Rho proteins are indirect processes via the GEFs and GAPs, respectively (Raftopoulou and Hall, 2004).

\subsection{Active and inactive forms of the small $G$ proteins}

We first develop a sub-model that describes a single small GTPase, in absence of crosstalk, keeping track of the active form bound to the plasma membrane, and the inactive form, that transits between membrane and cytosol. Let $G$ be the active form of a Rho protein, $G_{m}$ the inactive membrane-bound form, and $G_{c}$ the inactive cytosolic form. To avoid the obvious complication of distinct concentration units for the molecules on the membrane versus those in the cytosol, we will consider the protein levels as effective mean concentrations within a vertical column though the cell, assumed to be of constant thickness. We consider a thin slice through the cell (see Fig. 2). The domain for our spatial problem is the line, $0 \leq x \leq L$, where $L$ is a cell diameter. The boundaries are at the front and back of the cell. Since the proteins cannot diffuse out of the cell, it is appropriate to have no-flux boundary conditions at $x=0, L$.

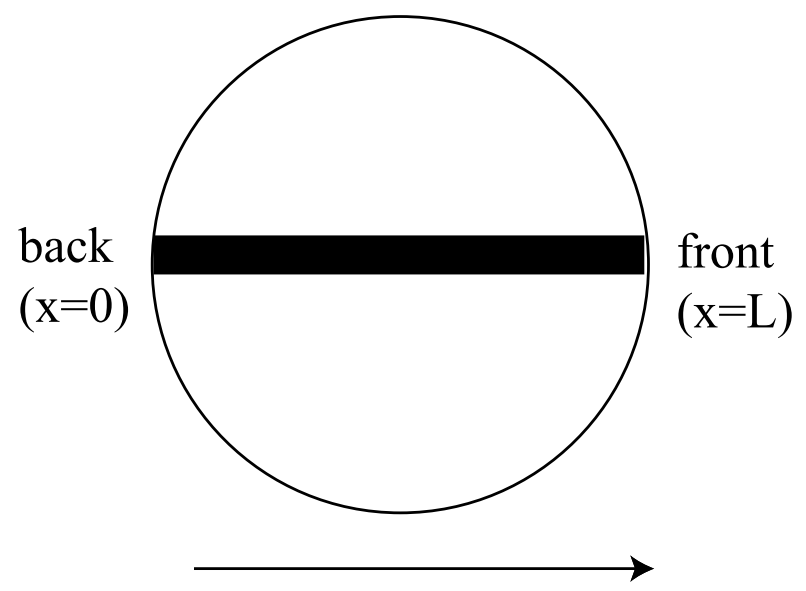

Fig. 2 Schematic representation of a moving cell. Arrow represents direction of movement. In one spatial dimension we interpret the boundary conditions as the front and back edges of the cell. 
On the timescale of interest, proteins are neither destroyed, nor synthesized, so that their total mean concentration is constant. On a (1D) domain, of length $L$,

$$
G_{\mathrm{tot}}=(1 / L) \int\left(G+G_{m}+G_{c}\right) d x
$$

Then

$$
\frac{\partial G}{\partial t}=k_{G}^{+} \mathrm{GEF}_{G} G_{m}-k_{G}^{-} \mathrm{GAP}_{G} G+D_{m} \Delta G
$$

where $k_{G}^{+}$and $k_{G}^{-}$are rates of activation and inactivation, and $D_{m}$ is the diffusion rate along the membrane. The inactive membrane bound and cytosolic forms of the protein satisfy the following equations:

$$
\begin{aligned}
\frac{\partial G_{m}}{\partial t} & =-k_{G}^{+} \mathrm{GEF}_{G} G_{m}+k_{G}^{-} \mathrm{GAP}_{G} G-k_{\mathrm{off}} G_{m}+k_{\mathrm{on}} G_{c}+D_{m} \Delta G_{m}, \\
\frac{\partial G_{c}}{\partial t} & =k_{\mathrm{off}} G_{m}-k_{\mathrm{on}} G_{c}+D_{c} \Delta G_{c}
\end{aligned}
$$

where $D_{c}$ is cytosolic diffusion, $k_{\text {off }}$ is the (GDI-mediated) membrane dissociation rate, and $k_{\text {on }}$ the membrane association rate (rate of GDI release). (This triplet of equations holds for each of the three GTPases, Cdc42, Rac, and Rho, creating a total of 9 equations. Further, as these proteins are approximately the same size and molecular weight, they share roughly equal values of $D_{m}, D_{c}$.)

Let $\hat{I}_{G}=k_{G}^{+} \mathrm{GEF}_{G} G_{\text {tot }}$ represent a basal rate of GEF-mediated activation; and let $\delta_{G}=k_{G}^{-} \mathrm{GAP}_{G}$ represent the basal inactivation rate mediated by GAPs. It is convenient to rescale the variables by the total amount of the given protein, so that, for example, $G_{m} / G_{\text {tot }}$ is the fraction of the total protein level (in a well-mixed system) that is in the inactive membrane-bound form locally. Then the equations can be written in the form

$$
\begin{aligned}
& \frac{\partial G}{\partial t}=\hat{I}_{G} \frac{G_{m}}{G_{\mathrm{tot}}}-\delta_{G} G+D_{m} \Delta G \\
& \frac{\partial G_{m}}{\partial t}=-\hat{I}_{G} \frac{G_{m}}{G_{\mathrm{tot}}}+\delta_{G} G-k_{\mathrm{off}} G_{m}+k_{\mathrm{on}} G_{c}+D_{m} \Delta G_{m}, \\
& \frac{\partial G_{c}}{\partial t}=k_{\mathrm{off}} G_{m}-k_{\mathrm{on}} G_{c}+D_{c} \Delta G_{c} .
\end{aligned}
$$

This system can be reduced by scaling arguments. Let $t^{*}=t D_{c} / L^{2}, x^{*}=x / L$, where $L$ is the length of the cell, and let $\Delta^{*}$ be the Laplacian in terms of the scaled spatial variable. Then (4c) becomes

$$
\frac{\partial G_{c}}{\partial t^{*}}=\frac{k_{\mathrm{off}} L^{2}}{D_{c}} G_{m}-\frac{k_{\mathrm{on}} L^{2}}{D_{c}} G_{c}+D_{c} \Delta^{*} G_{c} .
$$


Exchange of inactive GTPases between membrane and cytosol is very fast (Sako et al., 2000), so we assume that $\frac{k_{\mathrm{on}} L^{2}}{D_{c}}, \frac{k_{\mathrm{off}} L^{2}}{D_{c}} \sim O(1 / \varepsilon)$, where $\varepsilon$ is a small parameter. Then to the first approximation,

$$
0=-k_{\mathrm{on}} G_{c}+k_{\mathrm{off}} G_{m}, \quad \text { and } \quad G_{i}=G_{m}\left(1+\frac{k_{\mathrm{off}}}{k_{\mathrm{on}}}\right)=G_{c}\left(1+\frac{k_{\mathrm{on}}}{k_{\mathrm{off}}}\right) .
$$

We now replace the two forms, $G_{m}, G_{c}$, by a single inactive form $G_{i}=G_{c}+G_{m}$, where $G_{i}$ represents the total inactive GTPase level. Adding Eqs. (4b) and (4c) leads to

$$
\frac{\partial G_{i}}{\partial t}=\frac{\partial G_{m}}{\partial t}+\frac{\partial G_{c}}{\partial t}=-\hat{I}_{G} \frac{G_{m}}{G_{\mathrm{tot}}}+\delta_{G} G+D_{m} \Delta G_{m}+D_{c} \Delta G_{c} .
$$

Expressing $G_{m}, G_{c}$ in terms of $G_{i}$ using (6), leads to

$$
\frac{\partial G_{i}}{\partial t}=-I_{G} \frac{G_{i}}{G_{\mathrm{tot}}}+\delta_{G} G+D_{m c} \Delta G_{i}
$$

where $I_{G}=\hat{I}_{G} k_{\text {on }} /\left(k_{\text {on }}+k_{\text {off }}\right)$ and

$$
D_{m c}=\frac{k_{\mathrm{on}}}{k_{\mathrm{on}}+k_{\mathrm{off}}} D_{m}+\frac{k_{\mathrm{off}}}{k_{\mathrm{on}}+k_{\mathrm{off}}} D_{c} .
$$

This "effective diffusion coefficient", $D_{m c}$ is a weighted average of the membrane and cytosolic diffusion (where the weighting is the fraction of time spent on the membrane and in the cytosol). Since $D_{c} \gg D_{m}$, we have $D_{m c}>D_{m}$.

By the above reasoning, we have reduced the 9 PDE model of Eqs. (4) (for $G=$ $C, R, \rho)$, to a 6 PDE model consisting of three pairs of equations of the form

$$
\begin{aligned}
& \frac{\partial G}{\partial t}=I_{G} \frac{G_{i}}{G_{\mathrm{tot}}}-\delta_{G} G+D_{m} \Delta G, \quad \text { for } G=C, R, \rho, \\
& \frac{\partial G_{i}}{\partial t}=-I_{G} \frac{G_{i}}{G_{\mathrm{tot}}}+\delta_{G} G+D_{m c} \Delta G_{i}, \quad \text { for } G_{i}=C_{i}, R_{i}, \rho_{i} .
\end{aligned}
$$

The difference in diffusion coefficients proves to be significant. The activation rate $I_{G}$ depends on the level of $\mathrm{GEF}_{G}$, and therefore, indirectly, on the levels of other small GTPases (see below). We will later use the notation $f_{G}()$ to represent this assumed functional dependence.

\subsection{Inactive forms, $G_{i}$, viewed as parameters}

We first consider yet a further reduction of the system of Eqs. (10). By the relatively fast diffusion of the inactive forms, $G_{i}=C_{i}, R_{i}, \rho_{i}$, it follows that this system would rapidly reach a state in which these are homogeneously distributed in space. By comparison, the diffusion of the active forms $G=C, R, \rho$ is slow, and similar for all three GTPases.

We know that with the appropriate crosstalk, the system of GTPases polarizes, so that, for example, the active form of Cdc42 is high at the front, and low at the back of a motile cell. Meanwhile, the inactive form is close to homogeneously distributed. Now focus attention on a small slice at the front, and compare it to a small slice at the rear of a cell. 
Over these slices, there are effectively no gradients in the GTPases, so at these places, Eqs. (10) reduce to a set of ODEs,

$$
\frac{d G}{d t}=I_{G} \frac{G_{i}}{G_{\mathrm{tot}}}-\delta_{G} G, \quad \text { for } G=C, R, \rho,
$$

where $G_{i} \approx$ const. We idealize the experimentally observed spatial segregation of active Cdc42/Rac and Rho in migrating cells to mean that the cell maintains two stable zones with distinct levels of $\mathrm{Cdc} 42 / \mathrm{Rac}$ and Rho activity, corresponding to the front and back of the cell. Separating the front and back of the cell is a transition zone between these distinct (high versus low) active GTPase levels. With the exception of that narrow transition layer, we will consider the concentration of the Rho proteins at the front constant and different from the constant concentration in the back (illustrated in Fig. 4). In order to have two distinct values at front versus back, this system of 3 ODEs for the active forms of the Rho proteins should have multiple steady states, i.e., it should exhibit bistability once appropriate crosstalk terms are included in the GEF-mediated activation rates. Thus, for $G_{i}$ homogeneous in space (see Assumption 3), at any point in the domain away from the transition zone, the local dynamics of Cdc42, Rac, and Rho, are bistable. This idea will be helpful in arriving at the appropriate representation of the GTPase module.

It is important to comment that while $C_{i}, R_{i}, \rho_{i}$ are more or less homogeneous in space (due to rapid diffusion), they need not be constant in time. For example, suppose that the transition zone in active Cdc42 moves towards the back; this has to be at the expense of some of the inactive form of the protein. This would reduce $C_{i}$ everywhere in the cell, making it harder for further activation to occur. We will return to this point in due time, and show that this helps to stabilize transition zones and to avoid a common problem of traveling waves sweeping through the domain. It also means that bistability in Eqs. (11) does not imply that the full system is bistable as well. In fact, it is desirable for the full system to have a unique homogeneous steady state, because most cell types generally also have a stable rest state, with intermediate values of the small G-proteins. ${ }^{3}$

We next analyze the type of crosstalk terms in a modification of Eqs. (11) that leads to bistability as described above.

\section{Modeling crosstalk between the Rho proteins}

\subsection{Proposed interaction schemes}

A number of small GTPase crosstalk schemes have been proposed in the literature. These are summarized in Fig. 3. Some of these schemes include well-established interactions. Others contain hypothetical interactions or are incomplete. Our purpose here is to select the minimum complete set that can account for the observed phenomena. To do so, we make the following additional biologically reasonable assumptions:

\footnotetext{
${ }^{3}$ Even if the full system has only one homogeneous steady state, this does not automatically imply that the steady state is stable (corresponding to a stable rest state of the cell). In fact, for certain parameter settings, the homogeneous steady state can undergo a diffusion-driven instability related to Turing (see Appendix C). However, we will show that within a large parameter regime, the system can have both a stable rest state and a stable polarized state.
} 


\section{(a) Nobes and Hall (1995)}

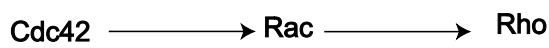

(c) Giniger (2002)
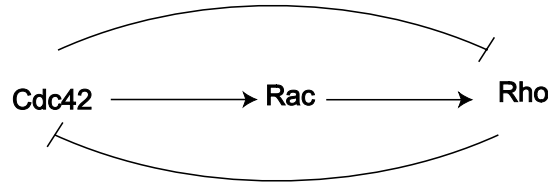

(e) van Leeuwen et al. (1997)

Cdc42

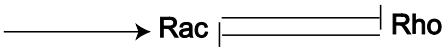

(g) Sakumura et al. (2005)

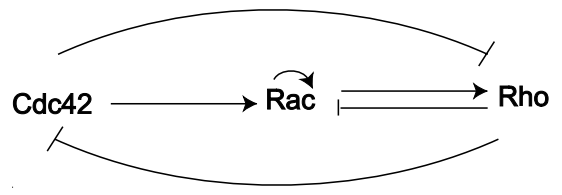

(b) Mackay and Hall (1998)

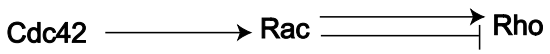

(d) Sander et al. (1999)

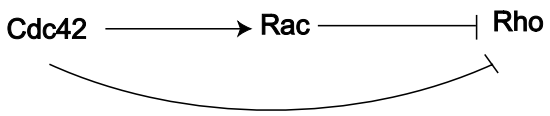

(f) Li et al. (2002)

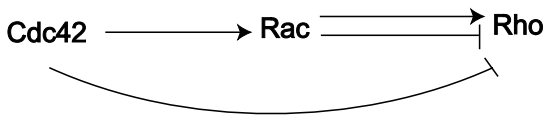

Fig. 3 Interactions between the Rho GTPases that have been proposed in the literature.

6. Crosstalk of Rho proteins occurs at the GTPase level via GEFs. Feedbacks between the Rho proteins means that activation rates can be concentration-dependent. The notion of 'activation' (respectively, 'inactivation') is taken to mean an up-regulation (respectively, downregulation) of a GEF. (This is based on the current literature, but see the Discussion for further comments.)

7. Cdc42 upregulates the activity of Rac, based on wide agreement in the published literature (Baird et al., 2005).

8. A system with bistable behavior for Cdc42-Rac-Rho should allow for switching between a [high level of Cdc42/Rac, low level of Rho] steady state and a [low level of Cdc42/Rac, high level of Rho] steady state, as described earlier. The simplest such system would have exactly three steady states. The two steady states (stable nodes/foci) would correspond to high Cdc42/Rac and low Rho level, and low Cdc42/Rac and high Rho level, respectively. The third state, a saddle point, would separate the basins of attraction of these two states.

As a consequence of Assumption 8, it is essential to have (either direct or indirect) feedback between Cdc42 and Rho in any scheme proposed for the small GTPase crosstalk. Thus, based on this fact alone, most schemes shown in Fig. 3 have to be incomplete.

For multi-stability to be possible, a positive feedback circuit is essential. (Alternately, an even number of negative interactions is equivalent to a positive feedback circuit.) Thomas (1981) had conjectured that this is a necessary condition for multiple steady states, and a number of proofs have been derived (Gouzé, 1998; Thomas and Kaufman, 


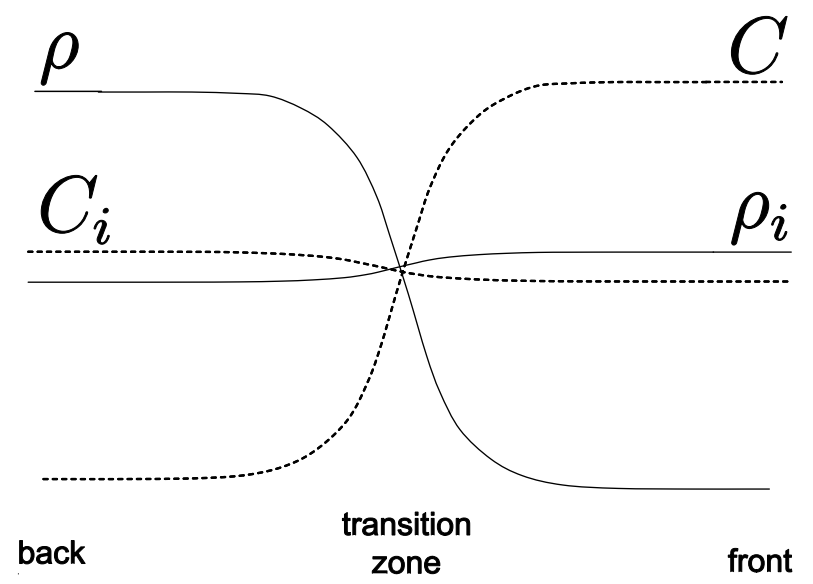

Fig. 4 A sketch of the pattern of distribution of the Rho proteins across the cell. Active Cdc42 $(C)$ and Rho $(\rho)$ are spatially bistable, forming distinct zones of activity. Rac follows the Cdc42 distribution (not shown). The distribution of the inactive forms, $C_{i}$ and $\rho_{i}$, is nearly homogeneous. Except for a transition zone, either Cdc42 level is high and Rho level is low (at the front), or Cdc42 level is low and Rho level is high (at the back). The actual transition zone is much narrower than shown in this diagram.

2001; Cinquin and Demongeot, 2002). Ferrell (2002) gives a nice overview of the biological consequences. Lacking any feedbacks on Cdc42 from Rac and Rho, the scheme in Fig. 3(a) does not, by itself, suffice for bistability and cannot account for observations. A similar problem occurs in the schemes in Figs. 3(b) and (d). The scheme in Fig. 3(e) has feedback between Rac and Rho, in the form of mutual antagonism, and can be shown to be consistent with bistability between these two intermediates. However, since Cdc42 receives no feedback, it would settle into a steady state that is independent of the distributions of Rac and Rho; this is contrary to experimentally measured gradients of active Cdc42 and Rac in moving cells. We dismiss this scheme. The scheme shown in Fig. 3(f) has the same defect as (e).

Since our system lacks a circuit with strictly positive feedbacks, an even number of negative feedback terms is needed in order to achieve a positive circuit required for multistability, as discussed above. Thus, for bistability of the Rho proteins it is necessary that two of the Rho proteins be inhibited via crosstalk. In particular, negative feedback must be present between $\mathrm{Cdc} 42$ and Rho. The only previously proposed schemes that have the type of negative feedback that could explain the bistable behavior required are shown in Figs. 3(c) and (g). In a review paper, Giniger (2002) suggested that scheme (c) could give rise to oscillatory behavior, without postulating further details of the crosstalk terms. Experiments consistent with scheme (c) appear in Benink and Bement (2005). Scheme (g), which was numerically investigated by Sakumura et al. (2005), is identical to (c), with the addition of direct inhibition of Rac by Rho (Rho also indirectly inhibits Rac via inhibition of Cdc42, which activates Rac) and an auto-catalytic activation for Rac. Sakumura et al. (2005) demonstrated that their extended scheme can give rise to oscillatory dynamics, and were not concerned with bistable behavior. Based on the above evidence, and in keeping with the principle to assume as little as possible, we select the simplest scheme, (c), as proposed by Giniger (2002). While other cases could possibly explain observations in the 
context of larger networks that may include indirect feedback from downstream effectors not explicitly considered here, we will concentrate on models that fall into case (c) or variants thereof.

\subsection{Including the crosstalk in the model equations}

Based on the arguments above, our 3 ODE version of the model for the active forms of the small GTPases has the form

$$
\begin{aligned}
& \frac{d C}{d t}=f_{C}(\rho)\left(\frac{C_{i}}{C_{\mathrm{tot}}}\right)-\delta_{C} C, \\
& \frac{d R}{d t}=f_{R}(C)\left(\frac{R_{i}}{R_{\mathrm{tot}}}\right)-\delta_{R} R, \\
& \frac{d \rho}{d t}=f_{\rho}(C, R)\left(\frac{\rho_{i}}{\rho_{\mathrm{tot}}}\right)-\delta_{\rho} \rho,
\end{aligned}
$$

with $C_{i}, R_{i}, \rho_{i}$ for the moment considered to be parameters. We now ask what types of functions (representing the effects of each of the proteins on one another's GEFs) would be consistent with bistability. In order to stay within the realm of plausible biochemical interactions, we make the following additional, biologically reasonable assumption:

9. Each protein is characterized by activation kinetics that include simple mass-action, Michaelis-Menten (saturated) or possibly cooperative sigmoidal (Hill-function) rates.

Table 1 lists the functions that are consistent with Assumption 9 and the crosstalk requirements

$$
\frac{\partial f_{C}}{\partial \rho}<0, \quad \frac{\partial f_{R}}{\partial C}>0, \quad \frac{\partial f_{\rho}}{\partial R}>0, \quad \frac{\partial f_{\rho}}{\partial C}<0 .
$$

In principle, any of the combinations shown in Table 1 would be appropriate for the functions $f_{G}(C, R, \rho)$. However, only a limited choice of functions can lead to the appropriate dynamical behavior.

To find the number of steady states for Eqs. (12), we set the set time derivatives to zero and solve the resulting system of algebraic equations. If all the functions $f_{G}(C, R, \rho)$ were linear, we would obtain a quadratic equation for the $\rho$ steady state with two possible

Table 1 Functional forms of activation/inactivation rates investigated for models based on the scheme in Fig. 5

\begin{tabular}{llll}
\hline Form & $\begin{array}{l}\text { Activation of Cdc42 } \\
\left(f_{C}(\rho)\right)\end{array}$ & $\begin{array}{l}\text { Activation of Rac } \\
\left(f_{R}(C)\right)\end{array}$ & $\begin{array}{l}\text { Activation of Rho } \\
\left(f_{\rho}(C, R)\right)\end{array}$ \\
\hline Linear & $I_{C}\left(1-\frac{\rho}{\rho_{\max }}\right)$ & $I_{R}+\alpha_{C} C$ & $\left(I_{\rho}+\alpha_{R} R\right)\left(1-\frac{C}{C_{\max }}\right)$ \\
Michaelis-Menten & $I_{C}\left(1-\frac{\rho}{k_{\rho}+\rho}\right)$ & $I_{R}+\frac{\alpha_{R} R}{k_{R}+R}$ & $\left(I_{\rho}+\frac{\alpha_{C} C}{k_{C}+C}\right)\left(1-\frac{C}{k_{C}+C}\right)$ \\
Hill function $(n>1)$ & $I_{C}\left(1-\frac{\rho^{n}}{k_{\rho}^{n}+\rho^{n}}\right)$ & $I_{R}+\frac{\alpha_{R} R^{n}}{k_{R}^{n}+R^{n}}$ & $\left(I_{\rho}+\frac{\alpha_{C} C^{n}}{k_{C}^{n}+C^{n}}\right)\left(1-\frac{C^{m}}{k_{C}^{m}+C^{m}}\right)$ \\
\hline
\end{tabular}




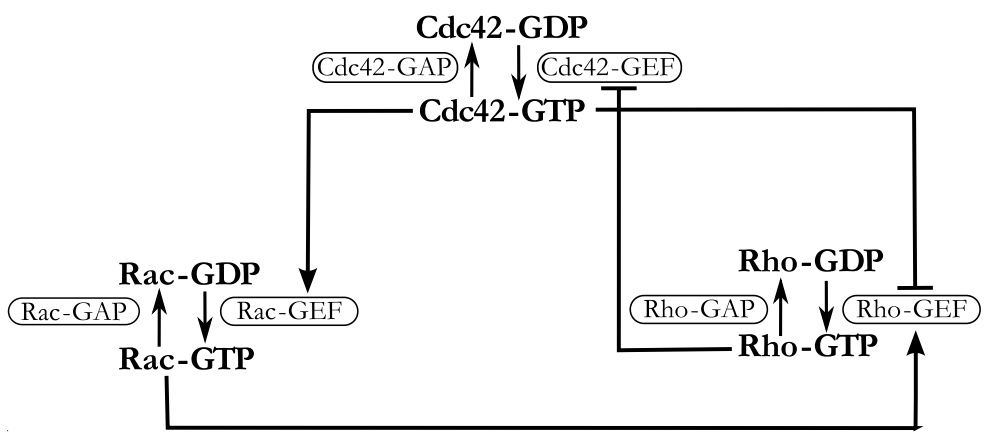

Fig. 5 Schematic diagram corresponding to the scheme we propose for the Rho protein crosstalk in Eqs. (17). GTP forms are active, GDP forms are inactive. Activation and inactivation are represented by vertical arrows and crosstalk is hypothesized to either up or down-regulate GTP-loading. Inhibition is hypothesized to occur due to downregulation of GTP-loading. This scheme is our realization of the Giniger crosstalk pathway shown in Fig. 3(c).

roots, each leading to a unique steady state value for $C$ and $R$. This is inconsistent with 3 possible steady states postulated by Assumption 8. Similarly, assuming Michaelian terms for inhibition and linear terms for activation, results in only a quadratic equation for one of the proteins, inconsistent with 3 equilibria. This means that without any cooperative term, there cannot be bistability in this system.

\subsection{Insights from a further reduction}

The experimentally observed mutual exclusion of Cdc42 and Rho suggested a further reduction for the purpose of gaining insight into the bistability, so we examined the 2 component system,

$$
\frac{d C}{d t}=g_{C}(\rho)-\delta_{C} C, \quad \frac{d \rho}{d t}=g_{\rho}(C)-\delta_{\rho} \rho,
$$

where $g_{G}=f_{G} G_{i} / G_{\text {tot }}$ for $G=C, \rho$. Nullclines of system (14), $d C / d t=0$ and $d \rho / d t=$ 0 , are just the curves $\delta_{C} C=g_{C}(\rho)$ and $\delta_{\rho} \rho=g_{\rho}(C)$ (where $g_{C}, g_{\rho}$ are continuous, positive functions of their arguments for $\rho \geq 0, C \geq 0$ ).

According to the mutual inhibition of Cdc42/Rho, $g_{\rho}^{\prime}(C)<0$ and $g_{C}^{\prime}(\rho)<0$, limiting the types of nullcline configurations. Further, these nullclines have to intersect three times in the positive $C-\rho$ quadrant, with one intersection at low $C$ high $\rho$, and another at low $\rho$ high $C$, both being stable steady states, separated by a saddle point. For a triple intersection, at least one of the nullclines has to have an S-shaped nonlinearity, and, for the appropriate stability, the direction of the crossing must be of the type shown in Fig. 6(a). Typical curves of this type were proposed in the "toggle switch" by Gardner et al. (2000). An appropriate biochemically relevant functional form for the Rho nullcline for such behavior is

$$
\rho=f_{\rho}(C) \approx I_{\rho}\left[1-\frac{C^{n}}{k_{\rho}^{n}+C^{n}}\right]=\frac{I_{\rho} k_{\rho}^{n}}{k_{\rho}^{n}+C^{n}}=\frac{I_{\rho}}{1+\left(C / k_{\rho}\right)^{n}},
$$




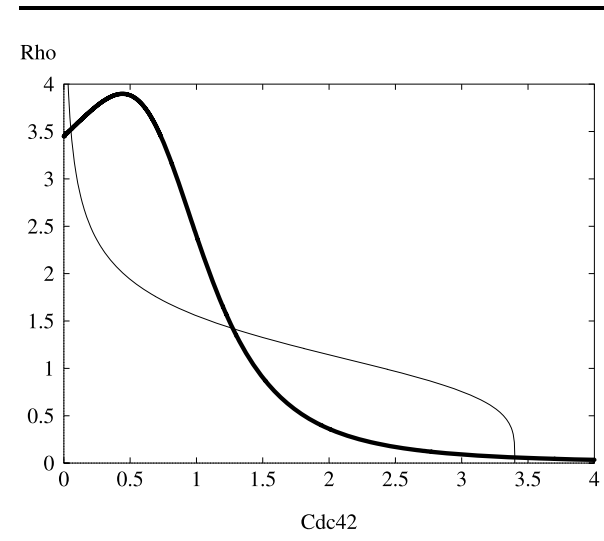

(a)
Rho

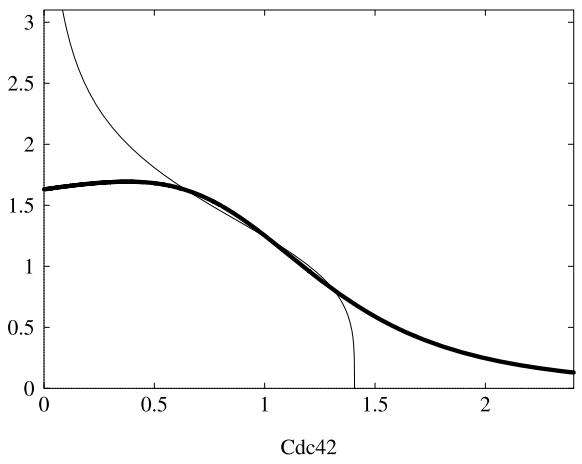

(b)

Fig. 6 (a) The $C-\rho$ phase plane (with $R$ on quasi-steady state) for Eqs. (17), showing two stable steady states separated by a saddle point. Nullclines shown are $\rho^{\prime}=0$ (thick curve) and $C^{\prime}=0$ (thin curve). Concentrations are measured in $\mu \mathrm{M}$. Here, we set $G_{i} / G_{\text {tot }}=1$, Hill coefficient $n=4$, and other parameter values as in Table 2. (b) Bistability also occurs if Eqs. (17) take into account limited availability of the inactive forms (using $G_{i}=G_{\text {tot }}-G$ for $G=C, R, \rho$ ), with the same parameters as in (a).

where $I_{\rho}$ is the basal Rho activation rate, as before, $n$ is a Hill coefficient and $k$ a "halfmaximal response" concentration. This function can be recognized as an activation rate that is decreased via cooperative reaction involving Cdc42 (or one of its downstream targets). Hill functions with $n \geqslant 2$ are commonly used to represent cooperativity in molecular phenomena. They can be derived by suitable approximation from the kinetics of multiple step reactions (see, for example, Edelstein-Keshet, 1988). The cooperativity could, for example, be realized through multi-step processes, GEF saturation, or stoichiometric inhibition of GEF activity (Ferrell, 1996).

A simple choice for the appropriate $C \rho$ model is

$$
\begin{aligned}
& \frac{d C}{d t}=\frac{I_{C} k_{C}^{m}}{k_{C}^{m}+\rho^{m}}\left(\frac{C_{i}}{C_{\text {tot }}}\right)-\delta_{C} C, \\
& \frac{d \rho}{d t}=\frac{I_{\rho} k_{\rho}^{n}}{k_{\rho}^{n}+C^{n}}\left(\frac{\rho_{i}}{\rho_{\mathrm{tot}}}\right)-\delta_{\rho} \rho,
\end{aligned}
$$

where at least one of $n, m>1$.

The analysis described here and in Section 3.2 leads to the following conclusions about bistability in a 2-component system driven by mutual inhibition: For multiple steady states, at least one of the interactions should have some degree of "cooperativity," i.e., a Hill coefficient greater than 1.

\subsection{The three component ODE system}

Note that Rac is not a passive participant in the establishment of Cdc42/Rho polarity; it has been established experimentally that changing the active Rac levels in a cell affects Rho levels (Ridley et al., 1992; Sander et al., 1999). Based on the two-component system (16), we arrive at the set of equations for active GTP-bound Cdc42, Rac, and Rho, 
consistent with the crosstalk scheme given in Giniger (2002). For simplicity, we chose a linear term, (e.g. $I_{R}+\alpha_{C} C$ ), to describe the activation of Rac by Cdc42 and of Rho by Rac. Our model ODE system is then

$$
\begin{aligned}
& \frac{\partial C}{\partial t}=\frac{I_{C}}{1+\left(\rho / \beta_{\rho}\right)^{n}}\left(C_{i} / C_{\mathrm{tot}}\right)-\delta_{C} C, \\
& \frac{\partial R}{\partial t}=\left(I_{R}+\alpha_{C} C\right)\left(R_{i} / R_{\mathrm{tot}}\right)-\delta_{R} R, \\
& \frac{\partial \rho}{\partial t}=\frac{\left(I_{\rho}+\alpha_{R} R\right)}{1+\left(C / \beta_{C}\right)^{n}}\left(\rho_{i} / \rho_{\mathrm{tot}}\right)-\delta_{\rho} \rho .
\end{aligned}
$$

Here $I_{G}=\hat{I}_{G} k_{\text {on }} /\left(k_{\text {on }}+k_{\text {off }}\right)$, as derived earlier $\left(\hat{I}_{C}, \hat{I}_{R}\right.$, and $\hat{I}_{\rho}$ are the basal activation rates in the absence of crosstalk). $\beta_{C}$ and $\beta_{\rho}$ describe levels of the proteins that lead to a half-maximal mutual inhibition of Cdc42 and Rho, respectively, and $\alpha_{C}$ and $\alpha_{\rho}$ are the activation of Rac by $\mathrm{Cdc} 42$, and the activation of Rho by Rac, respectively. $\delta_{C}, \delta_{R}$, and $\delta_{\rho}$ are the basal inactivation rates, as defined previously.

\subsection{Bistability in the ODEs}

The system of ODEs (17) can lead to bistability where either Cdc42 and Rac are high and Rho levels are suppressed, or Rho is high and Cdc42 and Rac are low. Figure 6(a) shows the nullclines for the well-mixed system in the case of $n=4$, with $R$ on quasisteady state (QSS), ${ }^{4}$ and using parameter values in Table 2 (see next section). For the $3 \mathrm{D}$ system it also holds that bistability can only occur if $n>1$ (Jilkine, 2005). Figure 7

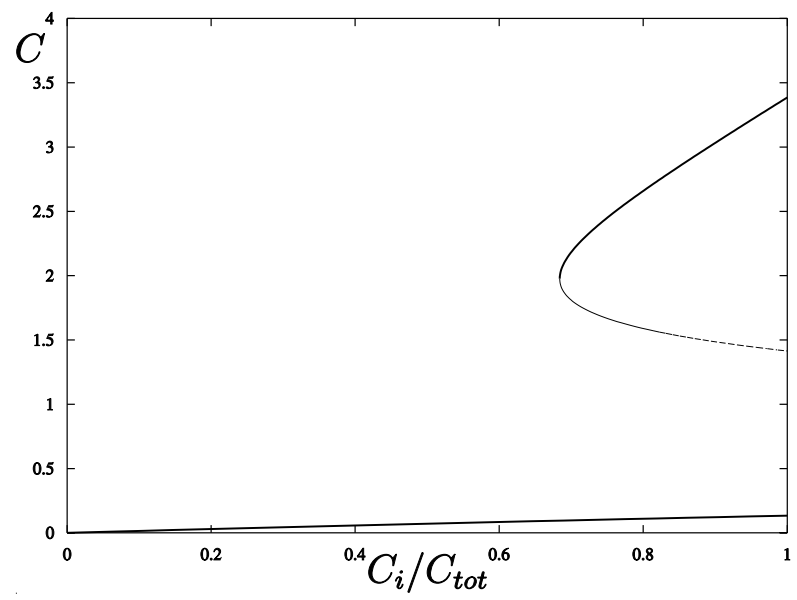

Fig. 7 A bifurcation diagram for Eqs. (17) for the parameter $C_{i} / C_{\text {tot }}$. All other values of $G_{i} / G_{\text {tot }}$ are set to $1, n=3$, and other parameter values are as given in Table 2. Note that when a large fraction of the protein is in the inactive form $\left(C_{i} / C_{\text {tot }} \geq 0.75\right)$, two stable steady states coexist.

\footnotetext{
${ }^{4}$ Note that a QSS assumption can never cause the loss of an equilibrium. Stability and dynamics, however, can change.
} 


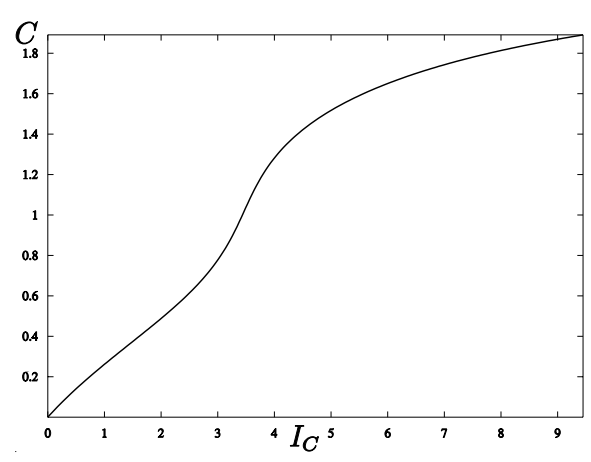

(a)

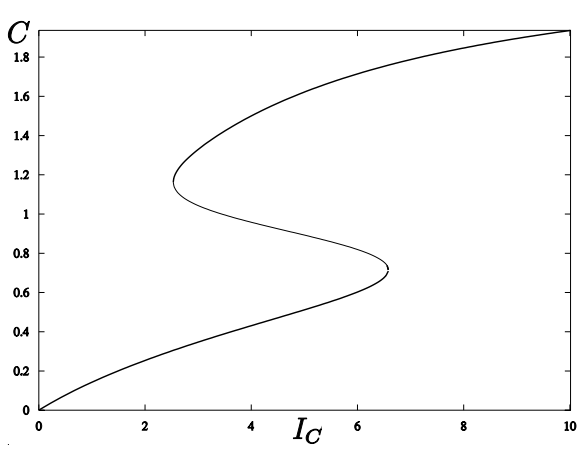

(b)

Fig. 8 Bifurcation diagrams for the Cdc42 activation rate, $I_{C}$ for $n=3$ (a) and $n=6$ (b) for Eqs. (17) including saturation due to inactive forms (letting $G_{i}=G_{\text {tot }}-G$ for $G=C, R, \rho$ ). Note that a higher Hill coefficient is necessary for bistability (compare with Fig. 7). Other parameter values used are given in Table 2 .

shows a bifurcation diagram for Eqs. (17) with $n=3$ and $0<C_{i} / C_{\text {tot }}<1$ treated as a parameter. This figure shows that a large fraction of the small G-proteins has to be in the inactive form, as has been observed experimentally (Boukharov and Cohen, 1998; Benard et al., 1999).

Figure 6(b) shows that, if instead of treating the inactive forms simply as parameters, we consider their limited availability (i.e., substitute $G_{i}=G_{\text {tot }}-G$ into Eqs. (17), it becomes difficult for the nullclines to cross each other three times, i.e., the region of bistability becomes very small. For the parameters given in Table 2, in the well-mixed system, bistability requires $n \geq 4$. If $n=6$, the bistable region becomes larger again (see Fig. 8), but, overall, for realistic parameter settings, global bistability is hard to find. This is actually a good property that prevents the cell as a whole from switching to one of the extremes.

A simplification of Eqs. (17) can be obtained by removing the first inhibitory term due to $\mathrm{Cdc} 42$ in the equation for $\rho$, i.e., replacing Eq. (17c) by

$$
\frac{d \rho}{d t}=\left(I_{\rho}+\frac{\alpha_{R} R}{1+\left(C / \beta_{C}\right)^{n}}\right)\left(\rho_{i} / \rho_{\mathrm{tot}}\right)-\delta_{\rho} \rho .
$$

The biological interpretation, that $\mathrm{Cdc} 42$ only inhibits the Rac-induced activation of Rho, could be reasonable. In this alternative version, Rho and Cdc42 still mutually suppress each other, and the resulting interactions are still sufficient for bistability. An experimental way to distinguish between Eqs. (17c) and (18) would be to see how increasing Cdc42 levels affects Rho in the case of a Rac knockout. If no effect is observed, Cdc 42 acts on Rho through a Rac-dependent pathway. On the other hand, if Rho levels decrease, we can conclude that Cdc42 inhibits GTP-loading of Rho in a Rac-independent manner.

No other simplifications of (17) will result in a biochemically plausible bistable system. So we conclude that the model given by Eqs. (17) is a minimal model of Cdc42, Rac, and Rho kinetics that gives bistable behavior as a result of mutual inhibition of $\mathrm{Cdc} 42$ and Rho via GEF repression. The model is consistent with biological evidence that points to the importance of the interactions between all three of the proteins, and in particular to 
mutual inhibition between the front and back (Xu et al., 2003). In Appendix A we show that interactions through GAPs could potentially explain cell polarization also, but that this still requires cooperativity in the reciprocal inhibition terms.

\section{Parameter estimates}

It is difficult to find detailed experimental data for many of the rate constants in the model. However, estimates can be made for the rates of decay and basal activation of the proteins. A summary of parameter values appears in Table 2.

The typical size of a eukaryotic cell is $10 \mu \mathrm{m}$ in diameter, so our domain is $0 \leq x \leq$ $10 \mu \mathrm{m}$. We based diffusion coefficients on estimates for heterotrimeric G-proteins in the membrane $\left(0.1 \mu \mathrm{m}^{2} \mathrm{~s}^{-1}\right)$ and cytosol $\left(10 \mu \mathrm{m}^{2} \mathrm{~s}^{-1}\right)$ (Postma et al., 2004).

According to Michaelson et al. (2001) (using immunoblotting in fibroblasts), the total amount of small G-proteins is 34,82 , and $26 \mathrm{ng} / 10^{6}$ cells, for $\rho, R$, and $C$, respectively. Based on the molecular weight of the small G-proteins ( $21 \mathrm{kDa}$, which is $\left.3.49 \times 10^{-11} \mathrm{ng}\right)$, and approximating cells as $10 \mu \mathrm{m}$ diameter spheres, this corresponds, respectively, to $(1.86,4.49$, and 1.42$)$ times $10^{3}$ molecules $/ \mu \mathrm{m}^{3}$. Using Avogadro's number $\left(6.02 \times 10^{23}\right)$, it follows that $1 \mu \mathrm{M}$ equals 602 molecules $/ \mu^{3}$, and therefore the effective total concentrations, i.e., $\rho_{\mathrm{tot}}, R_{\mathrm{tot}}$, and $C_{\mathrm{tot}}$, are 3.1, 7.5, and $2.4 \mu \mathrm{M}$.

Based on estimates of the fraction of small $\mathrm{G}$ proteins in the GTP-bound state (3\% up to 25\% for a resting cell) (Boukharov and Cohen, 1998; Benard et al., 1999) and the doubling of Rac and Cdc42 activity upon stimulation (Kurokawa et al., 2004), we took the overall fraction in the GTP-bound-state at steady state to be around $40 \%$ (i.e., $C \simeq 1$; $R \simeq 3 ; \rho \simeq 1.25 \mu \mathrm{M}$ ), and used these values as initial conditions unless stated otherwise. We further assumed that all saturation terms are in the same range as the typical steadystate concentrations, i.e., $\beta_{\rho}=1.25 \mu \mathrm{M} ; \beta_{C}=1 \mu \mathrm{M}$. A Hill coefficient of $n=4$ was used unless otherwise stated.

The average membrane lifetime of an activated Rac molecule is $2 \mathrm{~s}$ (Sako et al., 2000), giving a decay rate of $0.5 \mathrm{~s}^{-1}$. GAP-stimulated GTP hydrolysis of Rho has been measured

Table 2 Parameter estimates for the model

\begin{tabular}{lllll}
\hline Parameter & Meaning & Values & Units & Source \\
\hline$I_{C}$ & Cdc42 activation input rate & 3.4 & $\mu \mathrm{M} \mathrm{s}^{-1}$ & Section 4 \\
$I_{R}$ & Rac activation input rate & 0.5 & $\mu \mathrm{M} \mathrm{s}^{-1}$ & Section 4 \\
$I_{\rho}$ & Rho activation input rate & 3.3 & $\mu \mathrm{M} \mathrm{s}^{-1}$ & Section 4 \\
$\beta_{\rho}$ & Rho level for half-max inhibition of Cdc42 & 1.25 & $\mu \mathrm{M}$ & Section 4 \\
$\beta_{C}$ & Cdc42 level for half-max inhibition of Rho & 1 & $\mu \mathrm{M}$ & Section 4 \\
$n$ & Hill coefficient of Cdc42-Rho mutual & 4 & - & Sections 3.5, 4 \\
& $\quad$ inhibition response & & & \\
$\alpha_{C}$ & Cdc42-dependent Rac activation rate & 4.5 & $\mathrm{~s}^{-1}$ & Section 4 \\
$\alpha_{\rho}$ & Rac-dependent Rho activation rate & 0.3 & $\mathrm{~s}^{-1}$ & Section 4 \\
$\delta_{C}, \delta_{R}, \delta_{\rho}$ & Decay rates of activated small G-proteins & 1 & $\mathrm{~s}^{-1}$ & Sako et al. (2000) \\
$D_{m}$ & Membrane diffusion coefficient & 0.1 & $\mu \mathrm{m}^{2} \mathrm{~s}^{-1}$ & Postma et al. (2004) \\
$D_{m c}$ & Cytosolic diffusion coefficient & 10 & $\mu \mathrm{m}^{2} \mathrm{~s}^{-1}$ & Postma et al. (2004) \\
$C_{\text {tot }}$ & Effective total Cdc42 concentration & 2.4 & $\mu \mathrm{M}$ & Michaelson et al. (2001) \\
$R_{\text {tot }}$ & Effective total Rac concentration & 7.5 & $\mu \mathrm{M}$ & Michaelson et al. (2001) \\
$\rho_{\text {tot }}$ & Effective total Rho concentration & 3.1 & $\mu \mathrm{M}$ & Michaelson et al. (2001) \\
\hline
\end{tabular}


as $1.5 \mathrm{~s}^{-1}$ (Zhang and Zheng, 1998), so we took decay rates of the small G-proteins to be $\delta_{C}=\delta_{R}=\delta_{\rho}=1 \mathrm{~s}^{-1}$. Based on these estimated decay rates and approximate steady state concentrations, we inferred approximate activation rates for the small G-proteins, obtaining, $I_{C} \approx 3.4 \mu \mathrm{M} \mathrm{s}^{-1} ; I_{R}+1.0 \alpha \approx 5.0 \mu \mathrm{M} \mathrm{s}^{-1}$; and $I_{\rho}+3.0 \beta \approx 4.2 \mu \mathrm{M} \mathrm{s}^{-1}$. In our simulations, we use $I_{R}=0.5 \mu \mathrm{M} \mathrm{s}^{-1} ; \alpha_{R}=4.5 \mathrm{~s}^{-1} ; I_{\rho}=3.3 \mu \mathrm{M} \mathrm{s}^{-1}$; and $\alpha_{\rho}=0.3 \mathrm{~s}^{-1}$, to describe a strong dependency of Rac on $\mathrm{Cdc} 42$, and a weaker dependency of Rho on Rac.

\section{Spatial form of system (17)}

To avoid confusion about terminology, we define the following. Bistability means existence of two stable equilibria in the ODE model. Spatial bistability means that the PDE model exhibits zones of stable size, throughout which more or less constant concentration values are maintained. Polarity means spatial bistability with exactly two zones, a front and a back.

When the diffusion of the active GTP-bound Cdc42, Rac and Rho is included in the spatial variant of the model, we obtain the 3-PDE form shown below, where, as before, inactive forms, $C_{i}, R_{i}, \rho_{i}$, are viewed as parameters:

$$
\begin{aligned}
& \frac{\partial C}{\partial t}=f_{C}(\rho)\left(C_{i} / C_{\mathrm{tot}}\right)-\delta_{C} C+D_{m} \Delta C, \\
& \frac{\partial R}{\partial t}=f_{R}(C)\left(R_{i} / R_{\mathrm{tot}}\right)-\delta_{R} R+D_{m} \Delta R, \\
& \frac{\partial \rho}{\partial t}=f_{\rho}(C, R)\left(\rho_{i} / \rho_{\mathrm{tot}}\right)-\delta_{\rho} \rho+D_{m} \Delta \rho .
\end{aligned}
$$

where, henceforth,

$$
f_{C}(\rho)=\frac{I_{C}}{1+\left(\rho / \beta_{\rho}\right)^{n}}, \quad f_{R}(C)=\left(I_{R}+\alpha_{C} C\right), \quad f_{\rho}(C, R)=\frac{\left(I_{\rho}+\alpha_{R} R\right)}{1+\left(C / \beta_{C}\right)^{n}} .
$$

In Appendix B, we discuss the linear stability analysis of the above set of three PDEs to both uniform and spatially non-homogeneous perturbations. We show that the system with $G_{i} / G_{\text {tot }}$ as a parameter (where $G=C, R, \rho$ ) can have various instabilities, but does not have a diffusion-driven ("Turing") instability. That is, the addition of diffusion cannot in itself cause the system to become unstable unless it is already unstable (Small perturbations of a finite wavenumber will not grow via diffusive-type instability.) This result is not entirely unexpected. It is consistent with reaction-diffusion systems in two variables, where equal diffusion coefficients of interacting chemicals prevent Turing instability. (At the same time, we must recognize that when the dimension of interacting components in an RD system is enlarged, from 2 to 3 in this case, new phenomena can emerge, and previous conditions can become irrelevant.) Further, the linear stability analysis gives only "local" information about the dynamics. Indeed, in the next section, we show numerically that finite perturbations give rise to a graded distribution, not only when the homogeneous 
equilibrium is unstable, but even when it is (locally) stable. A sufficiently large perturbation can lead to the formation of an initially polarized pattern, i.e., the system has an inherent tendency to segregate. However, as discussed further on, this 3-PDE form of the model does not yet achieve a stable (longlived) spatial polarization.

Numerical exploration confirms that polarization of system (19) is ephemeral. Instead, for graded initial conditions we see the emergence of a traveling wave between the two stable equilibria, whose speed depends on the diffusion coefficient. This system resembles a class of models in which multiple steady states exist in a well-mixed system, but where no inhomogeneous stable pattern exists in the spatial form when diffusion of all components is equal (see Murray, 2002 for numerous examples).

The occurrence of traveling waves in our system is not surprising, in hindsight. We can connect this phenomenon to simpler examples that have been studied in the mathematical literature. For a single equation $u_{t}=\varepsilon^{2} u_{x x}+f(u)$, where $f(u)$ is bistable, a transition layer joining the two stable equilibria typically emerges given two-phase initial data (Carr and Pego, 1989). For a sufficiently small diffusion coefficient $D \approx \varepsilon^{2}$ this internal layer moves towards one of the spatially homogeneous equilibria, which are the only stable steady states of the system (Carr and Pego, 1989). This is related to the behavior in our system. In the next sections, we describe our numerical results, and then show how they are ameliorated by including dynamics of the inactive forms of the proteins.

\subsection{Numerical simulations of system (19)}

Numerical simulations of system (19) in a one-dimensional setting were carried out using the method of lines (Explicit Euler's method) and the software program XPP. ${ }^{5}$ We used a spatial step size of $\Delta x=0.1 \mu \mathrm{m}$. Runge-Kutta method of order 4 was used for solving the resulting discretized differential equations, with a time step of $\Delta t=0.005 \mathrm{~s}$.

Simulations were carried out with biologically based parameter values, given in Table 2, unless otherwise specified. For simplicity, the parameter $G_{i} / G_{\text {tot }}$ with $G=C, R, \rho$ was set to 1. In view of the similar sizes of the Rho proteins (Zerial and Huber, 1995), we took $D_{C}=D_{R}=D_{\rho}=D_{m}$. Because system (17) is bistable, the dynamics, and the eventual steady-state behavior depend on the initial conditions. We describe numerical simulations in which initial conditions were varied, and effects followed over a time period of 60 seconds.

With a spatially uniform initial condition of $C(x)=1.7 \mu \mathrm{M}, R(x)=17 \mu \mathrm{M}, \rho(x)=$ $2 \mu \mathrm{M}$, the system approaches the (spatially homogeneous) high Cdc42 steady state. Changing the initial conditions to $C(x)=1.5 \mu \mathrm{M}$ makes the system approach the high Rho steady state. A slight asymmetry in the initial Cdc42 distribution (a linear gradient from 1.5 to $1.6 \mu \mathrm{M}$ ) is amplified, and a spatial segregation of the small G-proteins arises (see Fig. 9). This distribution, however, is a slowly moving traveling wave that tends towards a spatially homogeneous high Cdc42 steady state after about 100 seconds. The speed of the wave depends on the diffusion coefficient $D_{m}$ (see Fig. 10). By changing the parameters, it is also possible to obtain a traveling wave sweeping towards the high Rho equilibrium. However, a stable spatially inhomogeneous steady state does not exist, as discussed in the previous section.

\footnotetext{
${ }^{5}$ The XPP package was developed by Bard Ermentrout of the University of Pittsburgh. It is available from http://www.math.pitt.edu/ bard/xpp/xpp.html.
} 


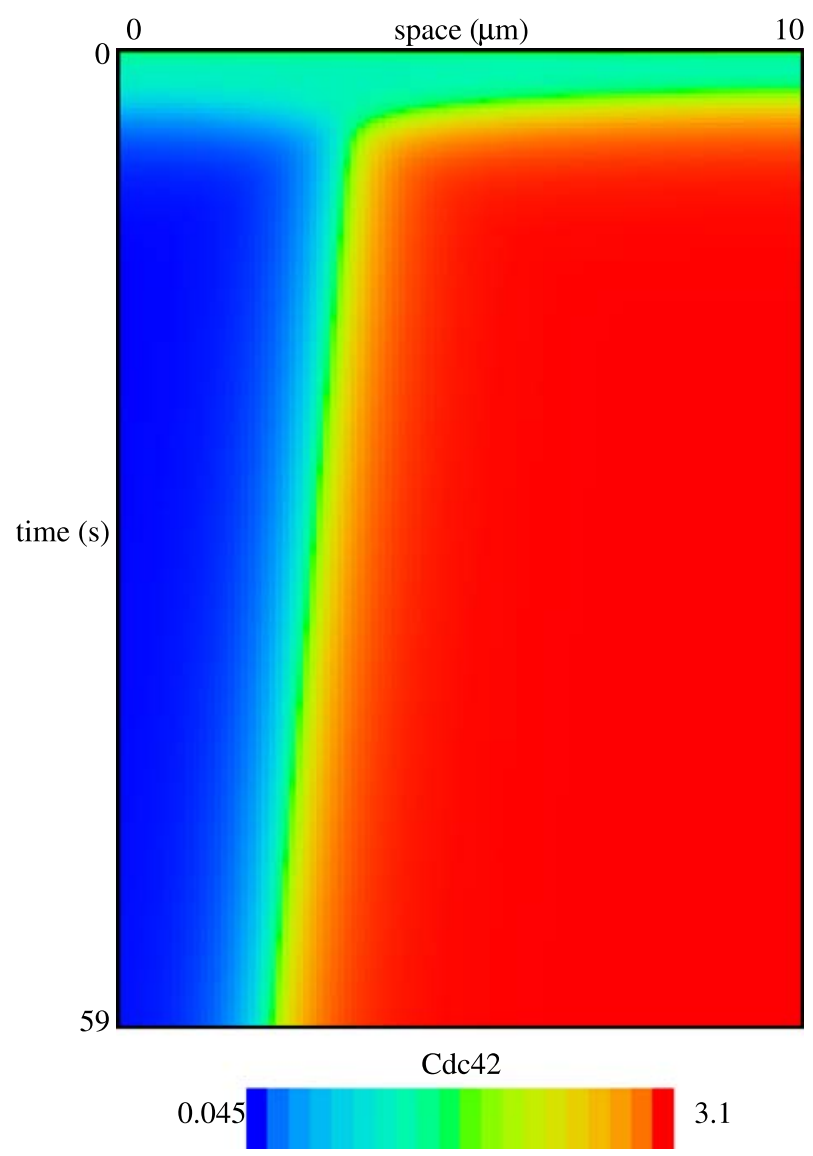

Fig. 9 Space-time plot of system (19). Time is on the vertical axis, space is on the horizontal axis. An initial gradient of active $\mathrm{Cdc} 42$ (1.5-1.6) leads to sharp spatial segregation of active Cdc42 and Rho. On a longer timescale (100 s) the system approaches a spatially homogeneous high Cdc42 steady state.

These results illustrate the fact that the active forms of the proteins on their own, even with mutual inhibition and crosstalk proposed in scheme (c), and even with bistability in the ODE formulation cannot yet account for the desired spatial polarization. What is missing is the stabilization of the transition zone between front and back. In the next section we show that incorporating the fast-diffusing inactive forms of the small GTPases brings the system to the desired dynamical behavior.

\section{The full (6 PDE) spatial model}

Thus far, we have treated the cytosolic pool of the small G-proteins as a limitless reservoir of available substrate. (The concentrations of the inactive cytosolic forms of the Rho proteins were taken as parameters.) Now returning to the full model that includes these 


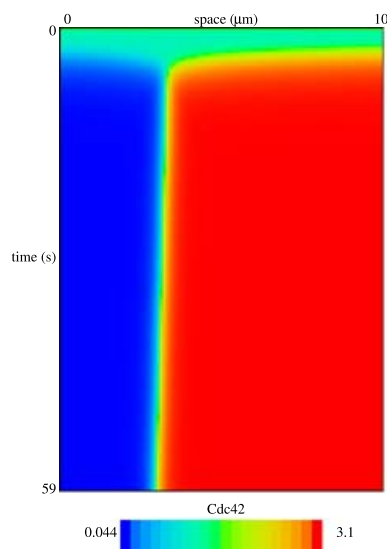

(a)

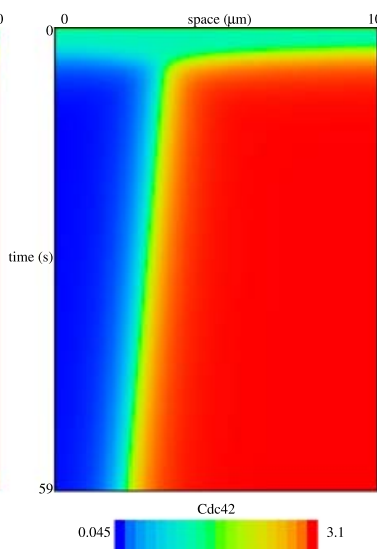

(b)

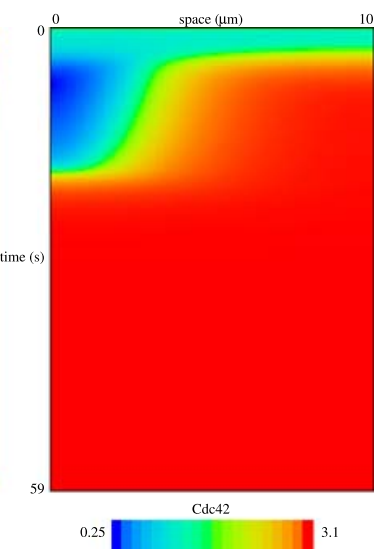

(c)

Fig. 10 The effect of the diffusion coefficient on the speed of the traveling wave in system (19). (a) $D_{m}=0.01 \mu \mathrm{m}^{2} \mathrm{~s}^{-1}$, (b) $D_{m}=0.1 \mu \mathrm{m}^{2} \mathrm{~s}^{-1}$, (c) $D_{m}=1 \mu \mathrm{m}^{2} \mathrm{~s}^{-1}$.

forms, we consider Eqs. (19), together with the fully dynamic set of equations for the inactive forms,

$$
\frac{\partial G_{i}}{\partial t}=-f_{G}+D_{m c} \Delta G_{i}, \quad \text { where } G=C, R, \rho
$$

The model consists of 6 PDEs, with crosstalk functions given in Eqs. (20), with no-flux boundary conditions, and parameters in Table 2 . The full set of equations is then

$$
\begin{aligned}
& \frac{\partial C}{\partial t}=f_{C}(\rho)\left(\frac{C_{i}}{C_{\mathrm{tot}}}\right)-\delta_{C} C+D_{m} \Delta C, \\
& \frac{\partial R}{\partial t}=f_{R}(C)\left(\frac{R_{i}}{R_{\mathrm{tot}}}\right)-\delta_{R} R+D_{m} \Delta C R, \\
& \frac{\partial \rho}{\partial t}=f_{\rho}(C, R)\left(\frac{\rho_{i}}{\rho_{\mathrm{tot}}}\right)-\delta_{\rho} \rho+D_{m} \Delta \rho, \\
& \frac{\partial C_{i}}{\partial t}=-f_{C}(\rho)\left(\frac{C_{i}}{C_{\mathrm{tot}}}\right)+\delta_{C} C+D_{m c} \Delta C_{i}, \\
& \frac{\partial R_{i}}{\partial t}=-f_{R}(C)\left(\frac{R_{i}}{R_{\mathrm{tot}}}\right)+\delta_{R} R+D_{m c} \Delta R_{i}, \\
& \frac{\partial \rho_{i}}{\partial t}=-f_{\rho}(C, R)\left(\frac{\rho_{i}}{\rho_{\mathrm{tot}}}\right)+\delta_{\rho} \rho+D_{m c} \Delta \rho_{i} .
\end{aligned}
$$

We investigated the linear stability analysis of these equations. In Appendix C, we show that under some parameter regimes, this system is capable of a diffusion-driven instability. In this case of six interacting components, the matrix manipulations becomes cumbersome. Therefore, we used the default parameter values and investigated the determinant 


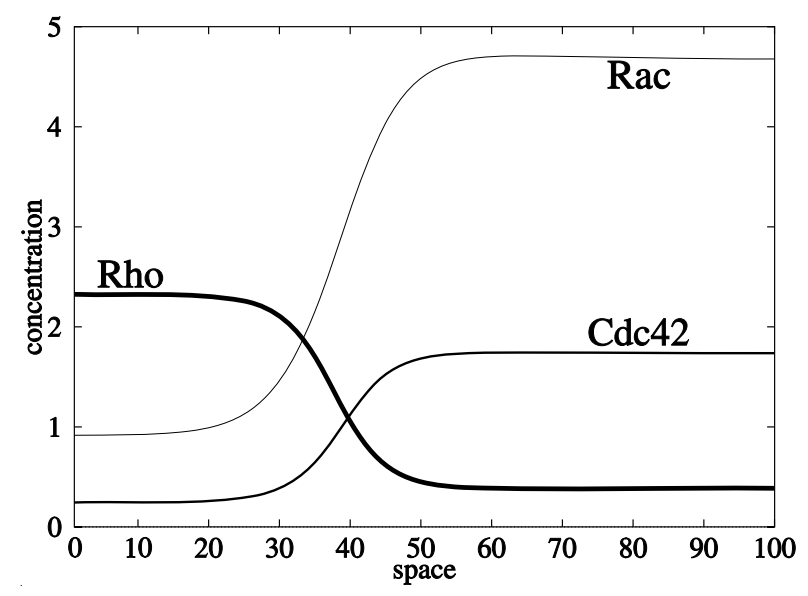

Fig. 11 Simulated steady state distribution of the system (19) with inactive forms given by Eqs. (21), and Hill coefficient $n=3$. Including dynamics of the inactive forms stabilizes a transition front, halting the traveling wave seen in Figs. 9 and 10. Other parameters are given in Table 2.

of the Jacobian numerically. (The instability is related to, but is not precisely the same as a generic Turing bifurcation as defined by Cross and Hohenberg, 1993. In our case, unstable wavenumbers are not strictly bounded away from 0 .) We found that there are wide parameter regimes where no diffusion-driven instability occurs. Nevertheless, a polarized pattern can be generated numerically, even in such regions, and, moreover, that pattern is stable once established, unlike the case of traveling waves in the previous 3-equation model. (See Fig. 11.)

We can understand part of this effect intuitively. The cytosolic diffusion of $C_{i}, R_{i}, \rho_{i}$ is fast, so these forms equilibrate rapidly over space. Binding and unbinding to the membrane is also fast, so these equilibrate between membrane and cytosol on a short timescale (Sako et al., 2000). For this reason, the inactive forms of the small GTPases rapidly carry global information. Low levels of the inactive forms in the cytosol imply overall high levels of activity. An equilibrium is rapidly established where expansion of the activated form of each of the small G-proteins is limited by the availability of the inactive form. The location of the transition zone is determined by the amount of the inactive form available. Flooding the cell with excess $C_{i}$ or $\rho_{i}$ causes the transition zone to shift, causing the high Cdc42 or high Rho equilibrium to take over the entire cell in some cases.

It should come as no surprise that a system of six nonlinear PDEs can have numerous types of dynamic behavior away from the homogeneous steady state(s). In this case, because an explicit form of the variables at the homogeneous steady state is not available due to the high degree of nonlinearity, producing a bifurcation diagram of the full 6-PDE system is challenging (but see Fig. C.3 and details in Appendices A-C for analysis based on the reduction that treats inactive forms of the Rho proteins as parameters.) Moreover, the detailed analysis of pinning of the traveling waves remains to be examined in the future using the tools of asymptotic analysis. It is a well known feature of non-local spatially distributed systems that wave-pinning and other effects can occur (Iron and Ward, 2000, 2001). 


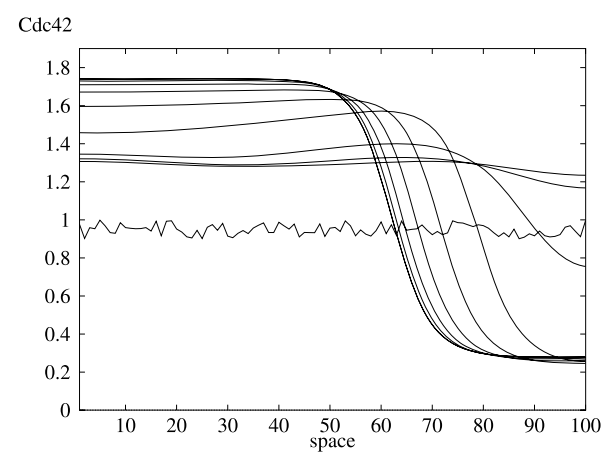

$\mathrm{Cdc} 42$

(a)

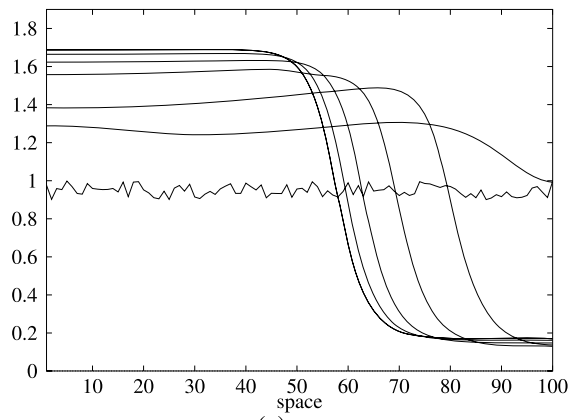

(c)

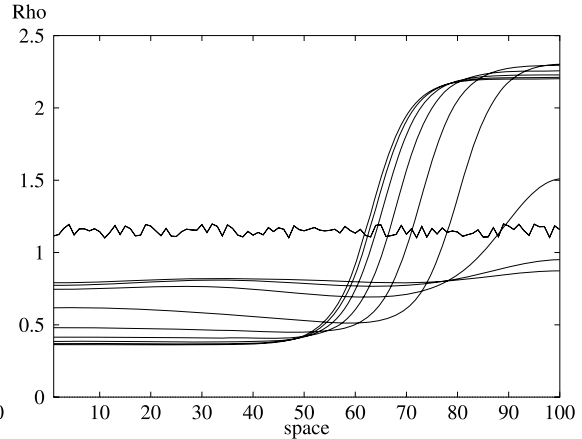

(b)

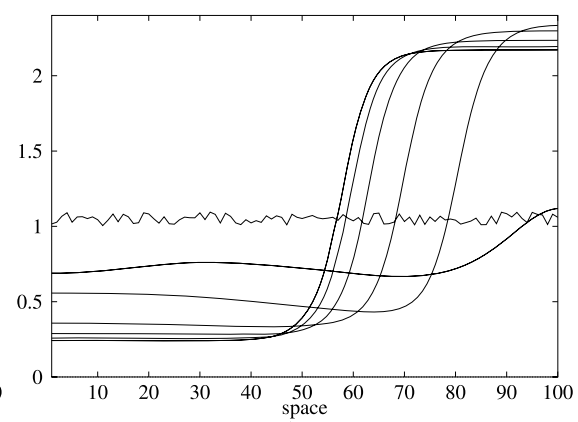

(d)

Fig. 12 Noisy initial conditions (no bias) result in spontaneous polarization of Cdc42 and Rho in system (19) with the inactive forms given by Eqs. (21). Spatial distributions of Cdc42 and Rho are shown from $t=0 \mathrm{~s}$ (initial random noise) until $t=90 \mathrm{~s}$, with intervals of $10 \mathrm{~s}$, during which the transition zone between Cdc42 and Rho moves from right to left. A steady state distribution is reached at around $t=90 \mathrm{~s}$ when $n=3$ and $60 \mathrm{~s}$ when $n=4$. Parameters as in Table 2. Hill coefficient: (a) $n=3$ (single homogeneous equilibrium); (b) $n=4$ (bistable regime).

\subsection{Numerical simulations of the full (6 PDE) system}

If the initial conditions and parameters for the Rho proteins in the extended system are spatially uniform, we obtain a spatially homogeneous distribution. However, random noise in the initial conditions (taken to be $C \simeq 1 ; R \simeq 3 ; \rho \simeq 1.25 \mu \mathrm{M}$, as discussed earlier) ensures that the system will spontaneously polarize (see Fig. 12). A small gradient in the initial conditions also leads to persistent spatial polarization (see Fig. 13). The resulting fronts are stable and not influenced by the magnitude of the gradient (see Fig. 13). Interestingly, the dynamics do not depend on whether the system has a single homogeneous equilibrium (Figs. 12, 13(a), (b)), or is in the bistable regime (Figs. 12, 13(c), (d)). In both cases persistent spatial polarization is found. This is surprising, because beforehand one would not expect polarization to occur when there is only one spatially homogeneous equilibrium, while in the bistable regime one would only expect to observe a transient polarization. Instead, independent of the bistability a persistent polarization is found. A transient gradient in the activation rate $I_{G}$ for one of the Rho proteins, which can be interpreted as a bias in the GEF distribution, will similarly lead to persistent polarity 


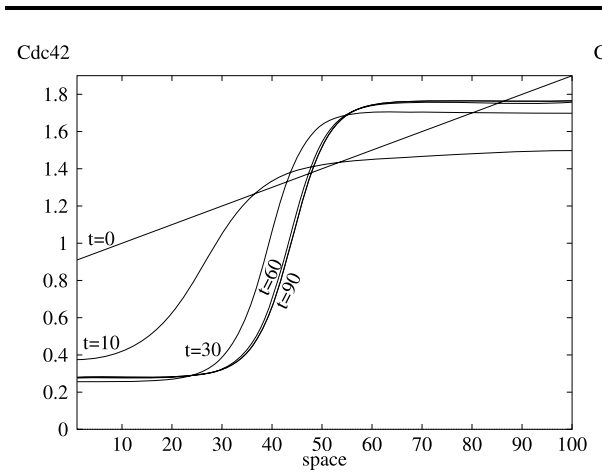

$\mathrm{Cdc} 42$

(a)

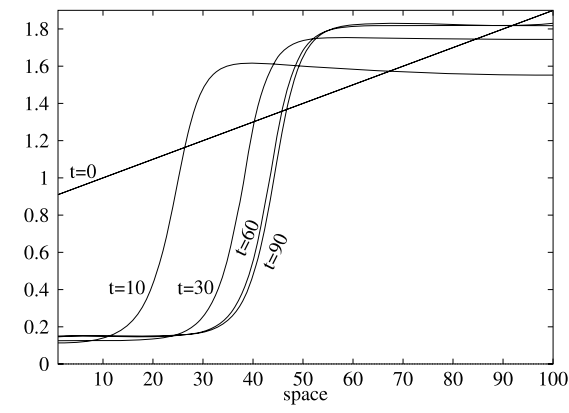

(c)

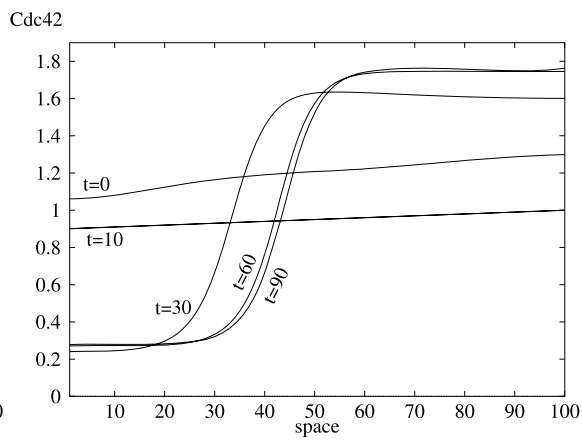

(b)

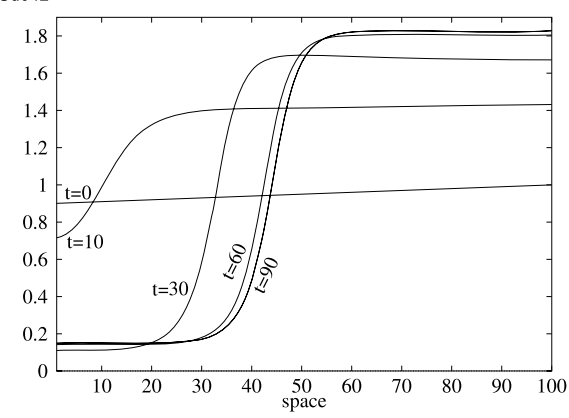

(d)

Fig. 13 Different initial stimuli (gradients of active Cdc42) lead to similar final steady state spatial distributions of active Rho GTPases. Spatial profiles for Cdc42 in the extended system (19) after 0, 10, 30, 60, and $90 \mathrm{~s}$, with the inactive forms given by Eqs. (21). Hill coefficient: (a), (b) $n=3$ (single homogeneous equilibrium); (c), (d) $n=4$ (bistable regime). Other parameters as given in Table 2. (a), (c) Initial Cdc42 gradient is $0.9-1.9 \mu \mathrm{M}$ across the cell. (b), (d) Initial Cdc42 gradient is $0.9-1.0 \mu \mathrm{M}$ across the cell. Steady state distributions are reached around $t=60 \mathrm{~s}$.

(see Marée et al., 2006). Thus, the system can respond to an externally imposed gradient, and can show spontaneous polarization. Note, however, that the system is still capable of approaching either of the spatially homogeneous equilibria if the initial conditions are sufficiently far away from $C \simeq 1 ; R \simeq 3 ; \rho \simeq 1.25 \mu \mathrm{M}$ that we used in the simulations.

Taken together, the numerical results of this and preceding variants of the model reveal that the crosstalk we have assumed, together with cooperativity in the kinetics can give rise to spatial polarization, and that the rapidly diffusing inactive forms of the Rho proteins play an important role in stabilizing the wavefront and leading to persistent polarity.

\section{Discussion}

The ability of a cell to sense and navigate up gradients of chemoattractants is known to have specific attributes, including amplification of very minute external signals, adaptation to a spatially uniform signal, rapid response to changes in the direction of the gradient, and the ability to spontaneously polarize (Parent and Devreotes, 1999). In recent years, a number of works have addressed the mechanisms responsible for spe- 
cific phenomena underlying chemotaxis. Such models are a vital tools in our evolving efforts to understand the mechanisms underlying chemotaxis (Ma et al., 2004; Subramanian and Narang, 2004).

Two distinct types of models have been proposed to explain gradient sensing. The first type explains the high amplification of the external gradient by a Turing type mechanism (Meinhardt, 1999; Postma and Van Haastert, 2001; Subramanian and Narang, 2004; Narang, 2006). Since the Rho proteins have very similar molecular weights, and hence similar rates of diffusion, it is not entirely surprising that the system comprising their active forms alone (i.e., our 3-variable PDE model) has no Turing instability. However, as noted in our modeling efforts, there are large differences in diffusion of membranebound and cytosolic forms. Recently, Narang (2006) developed a three component model (involving two mutually inhibiting activators and a global inhibitor) that gives rise to spatial segregation via a diffusion-driven instability mechanism. However, our full model (of three PDE's) achieves spatial segregation even in parameter regimes where we can show that no Turing instability is possible. Instead, depletion of substrate stabilizes a transition zone between the two equilibria. The location of this transition zone is determined by the available amount of the inactive form.

A different mechanism for gradient sensing was proposed by Levchenko and Iglesias (2002), and subsequently refined in Krishnan and Iglesias (2004) and Ma et al. (2004). In these models, based on "Local Excitation, Global Inhibition" (LEGI), the system remains sensitive to a changing external gradient, but loses polarity once the stimulus is removed. The LEGI module does not account for spontaneous polarization seen in motile cells (Wedlich-Soldner et al., 2003). The LEGI modules are informative for their dynamics, though they are not based on identifiable molecular components (as in the case of our model) (Ma et al., 2004).

A model for axon guidance based on crosstalk of the small GTPases by Sakumura et al. (2005) achieved gradient sensing by coupling Rho GTPase crosstalk to a "winner-takesdirection" decision point. For the small G-protein interactions, Sakumura et al. (2005) use a crosstalk scheme that is similar to ours. However, in our model, the Rho proteins inhibit one another through downregulation of the GEF that governs the basal activation (GTPloading) rate (see Fig. 5), while Sakumura et al. (2005) assume that mutual inhibition between Cdc42 and Rho passes through GAPs that govern the basal inactivation (GTPhydrolysis) rate. There is evidence in recent literature that in some cases, Rho crosstalk can be mediated by GEFs (Baird et al., 2005). However, basing a model on GEF or GAP mediated crosstalk is, at present, arbitrary, as there is no definitive evidence for one or the other. We show that for the bistability, cooperativity is needed in the mutual inhibition, whereas Sakumura et al. (2005) use Michaelis-Menten saturating terms. This is likely the reason why, despite using a similar crosstalk scheme, Sakumura et al. (2005) only find oscillations, while we achieve multi-stability.

It is important to note that crosstalk interactions we use in our model may not hold for all cell types. As we have shown in Fig. 3, alternate pathways have been proposed in the experimental literature. In particular, whether Rac activates or inhibits Rho seems to be dependent on cell type. In contrast to the activation term used in this study, inhibitory effects have, for example, been observed in neuronal cells and fibroblasts (Van Leeuwen et al., 1997; Sander et al., 1999). Especially in neutrophils, there is strong experimental evidence for the mutual inhibition between front and back (Xu et al., 2003; Wong et al., 2006). However, some recent evidence points to other, yet unknown, Rho 
family GTPases playing some of the roles in cell motility that were previously attributed to Cdc42 and Rac. Using a conditional knockout technique, Czuchra et al. (2005) and Vidali et al. (2006) found that Cdc42 and Rac, respectively, are not essential for migration in fibroblastoid cells, while Wheeler et al. (2006) found that Rac is not essential for macrophage migration. All these results suggest a high level of reduncancy within the small G-protein network. At the same time, when the same experimental technique was applied to neutrophils, it turned out that in those cells, Rac was actually necessary for chemotaxis (Sun et al., 2004). These examples show that there is still a high level of uncertainty with respect to the fine details the small G-protein interactions.

The spatiotemporal pattern of Rho activation also varies with cell type. While active Rho localizes to the back in chemotaxing neutrophils (Van Keymeulen et al., 2006), it has been observed at the front of randomly migrating fibroblasts (Pertz et al., 2006). Generally, it appears that mutual inhibition and spatial segregation of Rho GTPases occurs in fast moving cells that adhere loosely to the substratum (such as neutrophils). Slow moving, tightly adhering cells (such as fibroblasts) utilize a different mechanism for migration, which is yet to be understood. (Note that Rac-deficient fibroblasts migrate without a lamellipodium Vidali et al., 2006.)

Because our model focuses on the Rho proteins, an intermediate component in signal transduction to the cytoskeleton, we describe the extracellular chemoattractant gradient directly through a gradient of the active form (or alternatively GEF activity) along the membrane. The model shows extracellular gradient amplification (by translating it into a clear front and back, even when the gradient is very shallow), as well as cellular adaptation to increasing or decreasing chemoattractant levels (through the stabilizing size of the front and back). Due to the sheer number of potential GEFs and GAPs in the cell, ${ }^{6}$ it is unrealistic to develop detailed models linking specific GEFs/GAPs to the regulation of Rho protein activity at this time.

Although this paper deals only with the onset of polarization, we have also investigated Rho proteins and actin dynamics in a 2D model of a moving cell (Marée et al., 2006). We found that the Rho-protein "module" provides a robust mechanism for detecting a gradient and polarizing in response. With this module, the $2 \mathrm{D}$ cell remains sensitive to new external cues, and responds by turning. This is in accordance with neutrophils' ability to respond to a reversal of gradient by performing a U-turn (Xu et al., 2003).

One of the first responses of a cell to the chemotactic signal is the redistribution of the kinase PI3K, that produces $\mathrm{PIP}_{3}$, and the phosphatase, PTEN that degrades it, to the front and rear of the cell respectively (Iijima et al., 2002). This response is upstream of cytoskeletal rearrangement, since it occurs even in cells that have been subjected to actin depolymerizing drugs (Janetopoulos et al., 2004). However, it is not independent of the Rho proteins. The accumulation of $\mathrm{PIP}_{3}$ at the leading edge during polarization is known to be mediated by Rho GTPases through a positive feedback loop involving PI3K (Weiner et al., 2002; Srinivasan et al., 2003). Localization of PTEN is also regulated by Rho GTPases (Li et al., 2005). Our recent companion paper (Dawes and Edelstein-Keshet, 2007) uses the Rho-protein module developed here, links it to kinases, phosphatases, and

\footnotetext{
${ }^{6}$ There are about 60 mammalian GEFs which share a DH (Dbl homology) domain that catalyzes the exchange of guanine nucleotides, which is the active site for the GEF activity (Zheng, 2001); there are about eighty different proteins, containing a GAP-homology domain encoded in the human genome.
} 
lipids of the phosphoinositide module, and explores the synergy between these layers of signaling in generating the behavior of the motile cell.

By studying these additional layers of signaling, we found that many further properties of cell polarization could be explained; for example, we showed that phosphoinositides filter and smooth stimuli, and, so, prevent Rho proteins from forming multiple peaks in the domain in response to strong irregular stimuli (Dawes and Edelstein-Keshet, 2007). It is reasonable to speculate that further analysis of other signaling cascade components and layers, their internal crosstalk, and interactions between those layers will eventually reveal the modular mechanisms at play in the complex phenomena of cell polarization, and cell motility.

\section{Acknowledgements}

A.J. and L.E.K. have been funded by the Mathematics for Information Technology and Complex Systems (MITACS), and by a subcontract (to L.E.K.) from the National Science Foundation (USA) grant (number DMS-0240770) to Anders Carlsson, Washington University, St Louis. L.E.K. is also funded by the Natural Sciences and Engineering Research Council of Canada (NSERC). A.F.M.M. was supported by the Research Counsel for Earth and Life Sciences (ALW) with financial aid from the Netherlands Organization for Scientific Research (NWO).

\section{Appendix A Alternate 2-component model}

We carried out an analysis of a 2-component Cdc42-Rho system based on the assumption that each affects the other's rate of inactivation via GAPs instead of each other's rate of activation via GEFs, that is, we assumed that $I_{C}, I_{\rho}=$ const, $\delta_{C}=\delta_{C}(\rho), \delta_{\rho}=\delta_{\rho}(C)$. We considered the minimal bistable system in the form

$$
\begin{aligned}
& \frac{d C}{d t}=I_{C}-\delta(\rho) C, \\
& \frac{d \rho}{d t}=I_{\rho}-\delta(C) \rho .
\end{aligned}
$$

The nullclines are given by

$$
C=\frac{I_{C}}{\delta_{C}(\rho)}, \quad \rho=\frac{I_{\rho}}{\delta_{\rho}(C)} .
$$

Reasoning as before, both nullclines need to be decreasing functions of $C$. One of the nullclines, say $\rho=\frac{I_{\rho}}{\delta_{\rho}(C)}$, needs to be sigmoidal in shape in order for three steady states to exist. Using these constraints we obtain for the Rho nullcline

$$
\rho=\frac{I_{\rho}}{\delta_{\rho}(C)}=\frac{I_{\rho}}{k^{n}+C^{n}} \Rightarrow \delta_{\rho}(C)=k^{n}+C^{n},
$$


and for the $\operatorname{Cdc} 42$ nullcline

$$
\frac{d C}{d \rho}=-\frac{I_{c} \delta_{C}^{\prime}(\rho)}{\delta_{C}^{2}(\rho)}<0 \Rightarrow \delta_{C}^{\prime}(\rho)>0 .
$$

For simplicity choose $\delta_{C}(\rho)=\alpha \rho+$ constant, so the functional form for Eqs. (A.1) should be

$$
\begin{aligned}
& \frac{d C}{d t}=I_{c}-\alpha C \rho-\delta_{C} C, \\
& \frac{d \rho}{d t}=I_{\rho}-C^{n} \rho-\delta_{\rho} \rho .
\end{aligned}
$$

Based on the above reasoning we can conclude that to obtain the desired type of bistability in a two component mutually inhibitory system at least one of the interactions should have some "cooperativity" in order to get an S-shaped nullcline that intersects the other nullcline multiple times, as derived previously for inhibition through GEFs.

\section{Appendix B Linear stability analysis, inactive forms as parameters}

Here we consider the stability properties of PDEs of the models in this paper. We ask whether a Turing ("diffusive") instability can arise in these systems. Turing (1952) considered a special case of an activator-inhibitor system with distinct rates of diffusion, but here we have a larger set of intermediates for which the usual conditions for Turing patterns do not apply.

To avoid ambiguity in the terminology, we adopt the generalized terminology used in Cross and Hohenberg (1993) (see pp. 864, 870) for a Turing instability as a type $I_{s}$ (stationary periodic) instability: that is, where, according to linear stability analysis, a homogeneous steady state is stable to spatially uniform perturbations, but unstable to spatially non-uniform perturbations when diffusion is included. The main characteristic of such a system is that eigenvalues, $\sigma$ for growing perturbations have $\operatorname{Im}(\sigma)=0$ (i.e. no oscillations), and their corresponding most unstable wave-vectors (for $\operatorname{Re}(\sigma)>0$ ) have wavenumber $q_{0} \neq 0$ (i.e. emergence of standing wave patterns with fixed periodicity occurs at some bifurcation value of a parameter of interest).

As the full set of 6 PDEs is difficult to analyze, we first consider the set of 3 PDEs

$$
\begin{aligned}
& \frac{d C}{d t}=f_{C}(\rho)\left(\frac{C_{i}}{C_{\mathrm{tot}}}\right)-\delta C+D_{m} C_{x x}, \\
& \frac{d R}{d t}=f_{R}(C)\left(\frac{R_{i}}{R_{\mathrm{tot}}}\right)-\delta R+D_{m} R_{x x}, \\
& \frac{d \rho}{d t}=f_{\rho}(C, R)\left(\frac{\rho_{i}}{\rho_{\mathrm{tot}}}\right)-\delta \rho+D_{m} \rho_{x x},
\end{aligned}
$$


with $C_{i}, R_{i}, \rho_{i}$ taken as parameters. (Or, equivalently, a system in which there is an unlimited amount of inactive form, so that $G_{i} / G_{\text {tot }}=1$ for $G=C, R, \rho$.) Define

$$
\begin{array}{ll}
\left.\frac{C_{i}}{C_{\mathrm{tot}}} \frac{\partial f_{C}}{\partial \rho}\right|_{S S} \equiv-a_{1}<0, & \left.\frac{R_{i}}{R_{\mathrm{tot}}} \frac{\partial f_{R}}{\partial C}\right|_{S S} \equiv a_{2}>0, \\
\left.\frac{\rho_{i}}{\rho_{\mathrm{tot}}} \frac{\partial f_{\rho}}{\partial C}\right|_{S S} \equiv-a_{3}<0, & \left.\frac{\rho_{i}}{\rho_{\mathrm{tot}}} \frac{\partial f_{\rho}}{\partial R}\right|_{S S} \equiv a_{4}>0,
\end{array}
$$

where we have constructed all parameters above to be positive $\left(a_{i}>0\right)$, using the Giniger (2002) crosstalk scheme of Fig. 3(c). The set of linearized equations governing small perturbations about any homogeneous steady state are then

$$
\begin{aligned}
& \frac{d C}{d t}=-a_{1} \rho-\delta C+D_{m} C_{x x}, \\
& \frac{d R}{d t}=a_{2} C-\delta R+D_{m} R_{x x}, \\
& \frac{d \rho}{d t}=-a_{3} C+a_{4} R-\delta \rho+D_{m} \rho_{x x} .
\end{aligned}
$$

Consider spatially inhomogeneous perturbations of the form

$$
G(x, t)=G_{S S}+\epsilon_{G} e^{\sigma t} e^{i q x}, \quad G=C, R, \rho .
$$

Substituting these forms into the linearized PDEs and combining terms with like coefficients of $\epsilon$ leads to a system of 3 algebraic equations and to the Jacobian Matrix

$$
J=\left[\begin{array}{ccc}
\sigma+T & 0 & a_{1} \\
-a_{2} & \sigma+T & 0 \\
a_{3} & -a_{4} & \sigma+T
\end{array}\right]
$$

where we have defined

$$
T=\delta+D_{m} q^{2} \geq 0
$$

For nontrivial solutions, we require $\operatorname{det}(J)=0$, which leads to a cubic characteristic equation

$$
p(\sigma)=\sigma^{3}+\sigma^{2}(\underbrace{3 T}_{\alpha})+\sigma(\underbrace{3 T^{2}-a_{1} a_{3}}_{\beta})+(\underbrace{T^{3}-a_{1} a_{3} T+a_{1} a_{2} a_{4}}_{\gamma})=0 .
$$

We ask for conditions such that the homogeneous steady state is stable to spatially uniform perturbations $(q=0)$, but unstable to spatially nonuniform perturbations at some finite wave-number(s) $q_{\text {crit }}>0$. For this, we seek conditions such that $\operatorname{Re}(\sigma)<0$ for small $T$ (i.e. $T<T_{a}=\delta$ ), and $\operatorname{Re}(\sigma)>0$ for some larger value $T>T_{b}=D q^{2}+\delta$ (i.e. when diffusion is included). Restated, we look for a loss of stability as $T$ increases beyond some positive threshold value, $T_{b}$.

According to the Routh Hurwitz criterion, the conditions for $\operatorname{Re}(\sigma)<0$ are

$$
\alpha>0, \quad \gamma>0, \quad \alpha \beta-\gamma>0 .
$$


For the (generalized) Turing instability, these should all be satisfied if $D=0$ (for sufficiently small $T$ ), but one or more of these must be violated at some $T \geq T_{b}$ for instability to occur. The first condition, $\alpha>0$, is always true for positive $T$ values, so we ask how the second or third conditions can be violated as $T$ increases beyond a positive threshold. Defining

$$
\begin{aligned}
& \gamma=G(T)=T^{3}-a_{1} a_{3} T+a_{1} a_{2} a_{4}, \\
& \alpha \beta-\gamma=H(T)=8 T^{3}-2 a_{1} a_{3} T-a_{1} a_{2} a_{4},
\end{aligned}
$$

we note that $H(0)=-a_{1} a_{2} a_{4}<0$, and that $H(T)>0$ for $T$ sufficiently large. $H(T)$ changes sign in the direction of stability as $T$ is increased, so violating the condition $\alpha \beta-\gamma>0$ cannot lead to a diffusive instability.

Further, $G(0)=a_{1} a_{2} a_{4}>0$, and $G(T)>0$ for large $T$. The minimum value of $G$, attained at $T_{\min }=\sqrt{a_{1} a_{3} / 3}$ satisfies $H\left(T_{\min }\right)=-G\left(T_{\min }\right)$. We can dismiss the case that $G\left(T_{\min }\right) \geq 0$, which never leads to the desired diffusive instability. Noting that

$$
G(T)+H(T)=9 T^{3}-3 a_{1} a_{3} T=9 T\left(T^{2}-T_{\min }^{2}\right),
$$

we conclude that $G$ and $H$ cannot both be positive for any $0<T<T_{\min }$. This means that, even if $G\left(T_{\min }\right)<0, G$ has become negative for values of $T$ smaller than those at which $H$ becomes positive. Thus we cannot obtain instability by increasing $T$ beyond a positive threshold, ruling out instability of the Turing type in the above set of PDEs.

\section{Appendix C Linear stability of the full 6 PDE system}

We similarly investigate whether Turing type instability could occur in the full model, consisting of the 6 coupled PDEs for active and inactive GTPases:

$$
\begin{aligned}
& \frac{\partial C}{\partial t}=f_{C}(\rho)\left(\frac{C_{i}}{C_{\mathrm{tot}}}\right)-\delta_{C} C+D_{m} \Delta C, \\
& \frac{\partial R}{\partial t}=f_{R}(C)\left(\frac{R_{i}}{R_{\mathrm{tot}}}\right)-\delta_{R} R+D_{m} \Delta R, \\
& \frac{\partial \rho}{\partial t}=f_{\rho}(C, R)\left(\frac{\rho_{i}}{\rho_{\mathrm{tot}}}\right)-\delta_{\rho} \rho+D_{m} \Delta \rho, \\
& \frac{\partial C_{i}}{\partial t}=-f_{C}(\rho)\left(\frac{C_{i}}{C_{\mathrm{tot}}}\right)+\delta_{C} C+D_{m c} \Delta C_{i}, \\
& \frac{\partial R_{i}}{\partial t}=-f_{R}(C)\left(\frac{R_{i}}{R_{\mathrm{tot}}}\right)+\delta_{R} R+D_{m c} \Delta R_{i}, \\
& \frac{\partial \rho_{i}}{\partial t}=f_{\rho}(C, R)\left(\frac{\rho_{i}}{\rho_{\mathrm{tot}}}\right)+\delta_{\rho} \rho+D_{m c} \Delta \rho_{i} .
\end{aligned}
$$

In addition to previous notation for $a_{i}$, we also define

$$
\left.\frac{f_{C}(\rho)}{C_{\mathrm{tot}}}\right|_{S S} \equiv d_{1}>0,\left.\quad \frac{f_{R}(C)}{R_{\mathrm{tot}}}\right|_{S S} \equiv d_{2}>0,\left.\quad \frac{f_{\rho}(C, R)}{\rho_{\mathrm{tot}}}\right|_{S S} \equiv d_{3}>0,
$$


and $\delta_{C}=\delta_{R}=\delta_{\rho}=\delta$. The initial question is whether the model without diffusion is stable. For this, we consider the Jacobian:

$$
\left(\begin{array}{cccccc}
-\delta & 0 & -a_{1} & d_{1} & 0 & 0 \\
a_{2} & -\delta & 0 & 0 & d_{2} & 0 \\
-a_{3} & a_{4} & -\delta & 0 & 0 & d_{3} \\
\delta & 0 & a_{1} & -d_{1} & 0 & 0 \\
-a_{2} & \delta & 0 & 0 & -d_{2} & 0 \\
a_{3} & -a_{4} & \delta & 0 & 0 & -d_{3}
\end{array}\right)
$$

Since rows 4-6 are linearly dependent on rows $1-3$, the rank of the Jacobian matrix is 3 . This means that 3 out 6 eigenvalues are zero. Because the trace of the Jacobian, $(-3 \delta-$ $\left.d_{1}-d_{2}-d_{3}\right)$, is negative, at most two eigenvalues can be positive, depending on the values of $a_{1,2,3,4}$. If at least one eigenvalue is positive, the model is unstable to homogeneous perturbations, and leads to either multiple equilibria or oscillatory dynamics. When all eigenvalues of the above matrix are non-positive, the homogeneous equilibrium might still be unstable to spatially periodic perturbations, so we consider the model with diffusion.

We therefore consider solutions to the PDEs of the form

$$
v_{i}(x, t)=V_{i}(t) e^{i k x} .
$$

For ease of notation, define

$$
T_{m} \equiv k^{2} D_{m}, \quad b T_{m} \equiv T_{m c} \equiv k^{2} D_{m c}, \quad b=D_{m c} / D_{m},
$$

where $b$ is the ratio of the diffusion of the inactive forms to that of the active forms. Then the Jacobian now has new terms $-k^{2} D_{i}$ along its diagonal,

$$
\left(\begin{array}{cccccc}
-\delta-T_{m} & 0 & -a_{1} & d_{1} & 0 & 0 \\
a_{2} & -\delta-T_{m} & 0 & 0 & d_{2} & 0 \\
-a_{3} & a_{4} & -\delta-T_{m} & 0 & 0 & d_{3} \\
\delta & 0 & a_{1} & -d_{1}-b T_{m} & 0 & 0 \\
-a_{2} & \delta & 0 & 0 & -d_{2}-b T_{m} & 0 \\
a_{3} & -a_{4} & \delta & 0 & 0 & -d_{3}-b T_{m}
\end{array}\right) .
$$

The trace of the matrix is still negative, so the stability is solely determined by the sign of the determinant. Since it is now cumbersome to gain further analytical insights from this matrix, we investigate it numerically. We use default parameter settings (Table 2), but vary $T_{m}$ and $b$. Starting from the default value $b=100$ we find that for $0<T_{m}<0.418$, the determinant is negative (see Fig. C.1(a)), and thus the equilibrium is unstable to inhomogeneous perturbations. Since $D_{m}=0.1 \mu \mathrm{m}^{2} \mathrm{~s}^{-1}$, the value $T_{m}=0.418$ corresponds to a wavenumber $k=\sqrt{4.18}=2.044$, i.e., a wavelength of $2 \pi / k=3.072 \mu \mathrm{m}$, so any perturbation with a wavelength larger than $3 \mu \mathrm{m}$ grows. Figure C.1(b) shows the dependence of the largest eigenvalue, $\sigma_{\max }$ on $T_{m}$. The fastest growth rate (greatest value of $\sigma_{\max }$ ) occurs at $T_{m}=0.0441$, which corresponds to $9.46 \mu \mathrm{m}$. Indeed, numerical simulations over a length of $100 \mu \mathrm{m}$ show an initial increase of around 10 waves, corresponding to the most unstable mode. 


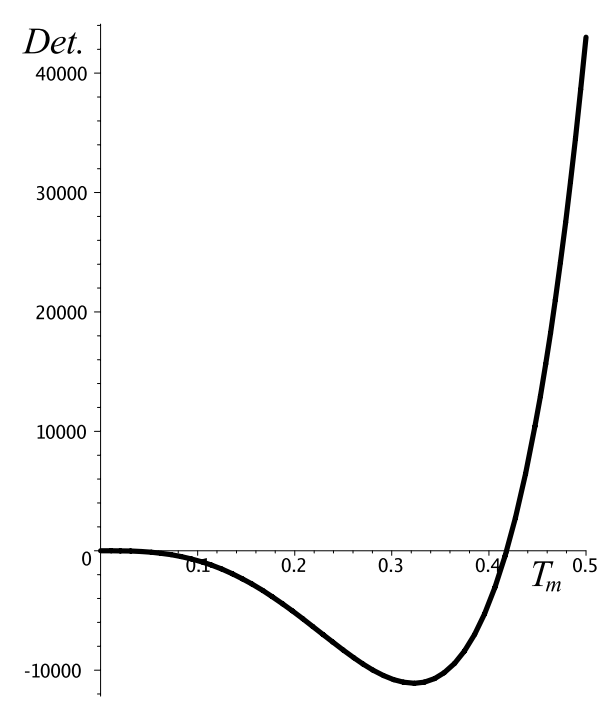

(a)

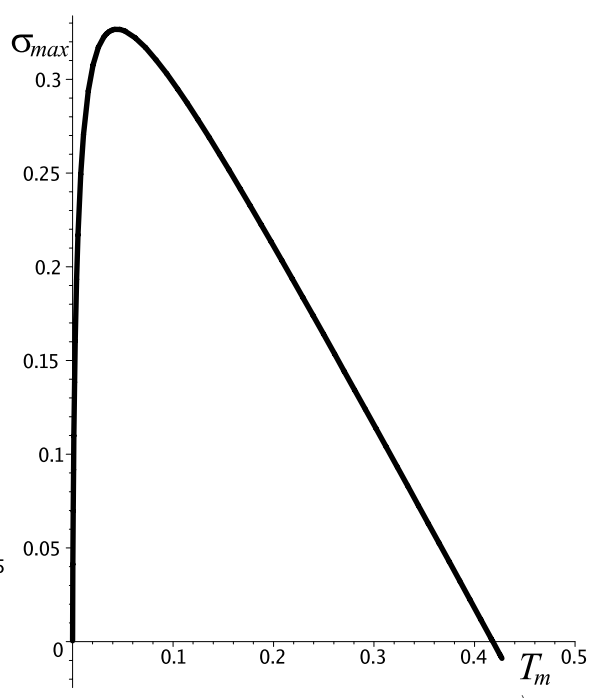

(b)

Fig. C.1 (a) The determinant of the Jacobian for the full (6 PDE) system as a function of $T_{m}=k^{2} D_{m}$ (for $k$ the wavenumber, $D_{m}$ the diffusion coefficient of active membrane-bound Rho proteins). $n=3$, all other parameter values are as given in Table 2. (b) Corresponding value of the largest positive eigenvalue, $\sigma_{\max }$, representing the fastest growing perturbation.

To determine the range of parameters for which the spatial model is unstable, we vary both $T_{m}$ and $b$, and plot the contour $\operatorname{Det}(J)=0$ in Fig. C.2. When $b>1.6$, (specifically, to the right and below this curve), $\operatorname{Det}(J)<0$ and we find unstable wavelengths. (Thus the value of $D_{m c}=b D_{m}$ has to be at least $0.16 \mu \mathrm{m}^{2} \mathrm{~s}^{-1}$ for instabilities to occur), with the bandwidth increasing when $b$ becomes larger. The graph saturates around $T_{m}=0.44$ when $b \approx 200$, limiting the highest unstable wavenumber.

Another observation to be made from Figs. C.1 and C.2 is that, regardless of the value of $b$, the bandwidth of unstable wavenumbers is always bounded by zero. This contrasts with the generic feature of Turing-type pattern formation, where dynamics are driven by a band of unstable wavenumbers bounded away from zero (Cross and Hohenberg, 1993). Consequently, in our system, very large wavelengths are unstable. This implies that in a very large cell ("large enough" for unstable modes to appear, e.g. if $b=D_{m c} / D_{m} \approx 2$, for example), the bipolar mode would be unstable.

The stability analysis of the full 6 PDE system and the stability analysis of the reduced system in which the levels of the inactive forms of Rho proteins are treated as parameters are actually closely linked to one another. For the inactive forms, if $D_{m c} \rightarrow \infty$, for any choice of $k$, the perturbation $v_{i}(x, t) \rightarrow 0$ for $t>0$, since the infinitely fast diffusion immediately erases any applied perturbation. Restated, treating the levels of inactive Rho proteins as fixed parameter values corresponds to analysing the limit $D_{m c} \rightarrow \infty$. We show this limit in the bifurcation diagram of Fig. C.3.

The black line in Fig. C. 3 shows the homogeneous equilibrium $\mathrm{Cdc} 42$ value as a function of $I_{C}$. This is equivalent to the line in Fig. 8(a) corresponding to a unique homogeneous equilibrium. (To obtain this curve, the inactive forms were fixed at the equilibrium 


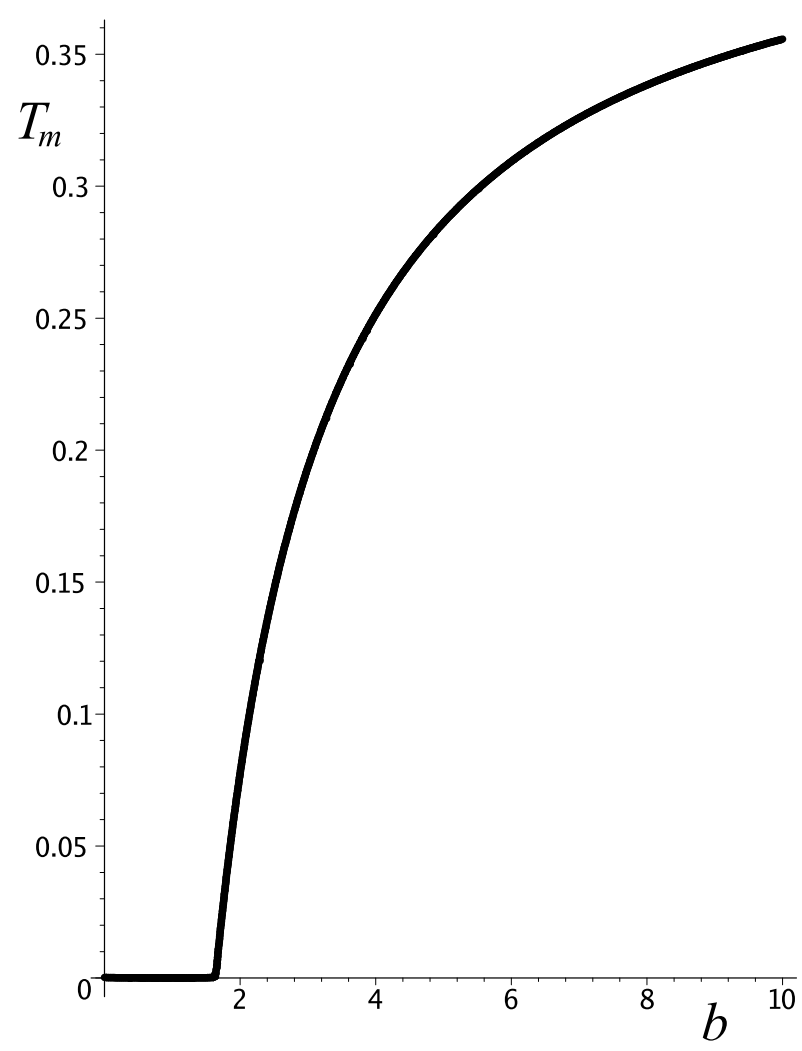

Fig. C.2 A plot of the $\left(T_{m}, b\right)$ parameter plane showing the contour along which $\operatorname{Det}(J)=0$ for the full (6 PDE) system. The parameter $b=D_{m c} / D_{m}$ is the ratio of diffusion coefficients of inactive to active Rho proteins. When $b>1.6$, there are unstable wavelengths, with the bandwidth increasing when $b$ becomes larger. The graph saturates around $T_{m}=0.44$ when $b \approx 200$.

value corresponding to the homogeneous steady state, which varies with $I_{C}$.) In this limit of inactive forms treated as parameters, we find that between $2.64<I_{C}<5.27$ the homogeneous equilibrium is unstable: within this interval, for sufficiently large values of $D_{m c}$, spatially inhomogeneous perturbations would grow. Outside this region, the homogeneous equilibrium is stable to both homogeneous and periodic perturbations for any choice of diffusion rates (as demonstrated in Appendix B). Accordingly, for $I_{C}<2.64$ or $I_{C}>5.27$, there are no combinations of the parameters $T_{m}$ and $b$ that result in $\operatorname{Det}(J)<0$, so small spatial inhomogeneities would decay.

The grey line in Fig. C.3 corresponds to other equilibria that are found when the inactive forms are kept fixed at these homogeneous steady state values. When such an equilibrium is stable, it means that a small, narrow perturbation (on a sufficiently small spatial scale, so as not to change the levels of the inactive forms) is able to establish itself. Thus, stable regions along the grey line correspond to directly reachable heterogeneous equilibria. For values of $I_{C}>1.86$, we always find three equilibria, two of which are stable. Consequently, a sufficiently large stimulus would lead to an initial polarization, followed 


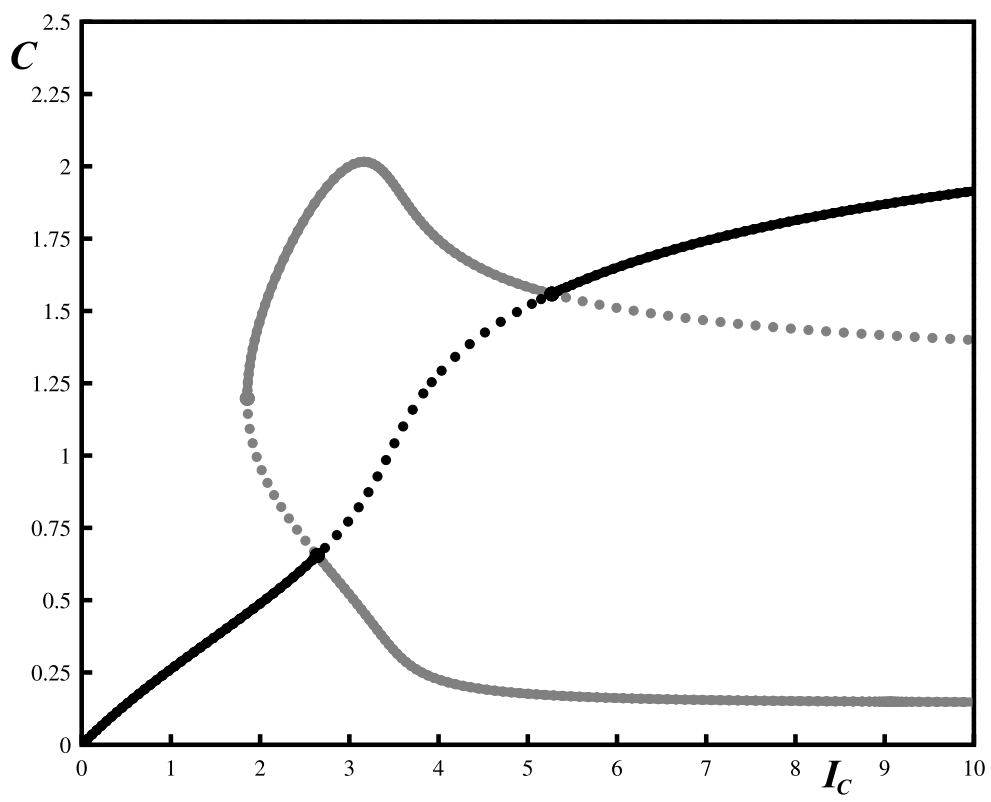

Fig. C.3 Bifurcation analysis of the reduction treating the levels of inactive forms of Rho proteins as parameters. The black line corresponds to the homogeneous equilibrium, the grey line corresponds to directly reachable heterogeneous equilibria. Between $2.64<I_{C}<5.27$, the homogeneous equilibrium is unstable, i.e., for sufficiently large values of $D_{m c}$, spatially inhomogeneous perturbations would grow. Outside of this region, the homogeneous equilibrium is stable to both homogeneous and periodic perturbations for any choice of diffusion rates. For values of $I_{C}>1.86$, there are three equilibria, two of which are stable. Consequently, a sufficiently large stimulus would always lead to an initial polarization.

by an expansion and retraction of different zones. During the latter process, the levels of the inactive forms change gradually, until an equilibrium is reached (described in Section 6). Between $2.64<I_{C}<5.27$ any signal is sufficient to trigger polarization, while outside this interval, the perturbation has to be sufficiently large to cause a pattern to form.

\section{References}

Baird, D., Feng, Q., Cerione, R.A., 2005. The Cool-2/ $\alpha$-Pix protein mediates a Cdc42-Rac signaling cascade. Curr. Biol. 15, 1-10.

Benard, V., Bohl, B.P., Bokoch, G.M., 1999. Characterization of Rac and Cdc42 activation in chemoattractant-stimulated human neutrophils using a novel assay for active GTPases. J. Biol. Chem. 274, 13198-13204.

Benink, H.A., Bement, W.M., 2005. Concentric zones of active RhoA and Cdc42 around single cell wounds. J. Cell Biol. 168, 429-439.

Bishop, A.L., Hall, A., 2000. Rho GTPases and their effector proteins. Biochem. J. 348, 241-255.

Boukharov, A.A., Cohen, C.M., 1998. Guanine nucleotide-dependent translocation of RhoA from cytosol to high affinity membrane binding sites in human erythrocytes. Biochem. J. 330, 1391-1398.

Caron, E., 2003. Rac signalling: a radical view. Nat. Cell Biol. 5, 185-187.

Carr, J., Pego, R.L., 1989. Metastable patterns in solutions of $u_{t}=\varepsilon^{2} u_{x x}-f(u)$. Comm. Pure Appl. Math. 42, 523-576. 
Cinquin, O., Demongeot, J., 2002. Positive and negative feedback: striking a balance between necessary antagonists. J. Theor. Biol. 216, 229-241.

Cross, M.C., Hohenberg, P.C., 1993. Pattern formation outside of equilibrium. Rev. Mod. Phys. 65, 8511112.

Czuchra, A., Wu, X., Meyer, H., Van Hengel, J., Schroeder, T., Geffers, R., Rottner, K., Brakebusch, C., 2005. Cdc42 is not essential for filopodium formation, directed migration, cell polarization, and mitosis in fibroblastoid cells. Mol. Biol. Cell 16, 4473-4484.

Dawes, A.T., Edelstein-Keshet, L., 2007. Phosphoinositides and rho proteins spatially regulate actin polymerization to initiate and maintain directed movement in a one-dimensional model of a motile cell. Biophys. J. 92, 744-768.

DerMardirossian, C., Schnelzer, A., Bokoch, G.M., 2004. Phosphorylation of RhoGDI by Pak1 mediates dissociation of Rac GTPase. Mol. Cell 15, 117-127.

Edelstein-Keshet, L., 1988. Mathematical Models in Biology. Random House, New York.

Evers, E.E., Zondag, G.C.M., Malliri, A., Price, L.S., Ten Klooster, J.-P., Van Der Kammen, R.A., Collard, J.G., 2000. Rho family proteins in cell adhesion and cell migration. Eur. J. Cancer 36, 1269-1274.

Ferrell, J.E., Jr., 1996. Tripping the switch fantastic: how a protein kinase cascade can convert graded inputs into switch-like outputs. Trends Biochem. Sci. 21, 460-466.

Ferrell, J.E., Jr., 2002. Self-perpetuating states in signal transduction: positive feedback, double-negative feedback and bistability. Curr. Opin. Cell Biol. 14, 140-148.

Gardner, T.S., Cantor, C.R., Collins, J.J., 2000. Construction of a genetic toggle switch in Escherichia coli. Nature 403, 339-342.

Giniger, E., 2002. How do Rho family GTPases direct axon growth and guidance? A proposal relating signaling pathways to growth cone mechanics. Differentiation 70, 385-396.

Gouzé, J.-L., 1998. Positive and negative circuits in dynamical systems. J. Biol. Syst. 6, 11-15.

Iijima, M., Huang, Y.E., Devreotes, P., 2002. Temporal and spatial regulation of chemotaxis. Dev. Cell 3, 469-478.

Iron, D., Ward, M.J., 2000. A metastable spike solution for a nonlocal reaction-diffusion model. SIAM J. Appl. Math. 60, 778-802.

Iron, D., Ward, M.J., 2001. Spike pinning for the Gierer-Meinhardt model. Math. Comput. Simul. 55, 419-431.

Itoh, R.E., Kurokawa, K., Ohba, Y., Yoshizaki, H., Mochizuki, N., Matsuda, M., 2002. Activation of Rac and $\mathrm{Cdc} 42$ video imaged by fluorescent resonance energy transfer-based single-molecule probes in the membrane of living cells. Mol. Cell. Biol. 22, 6582-6591.

Janetopoulos, C., Ma, L., Devreotes, P.N., Iglesias, P.A., 2004. Chemoattractant-induced phosphatidylinositol 3,4,5-trisphosphate accumulation is spatially amplified and adapts, independent of the actin cytoskeleton. Proc. Natl. Acad. Sci. U.S.A. 101, 8951-8956.

Jilkine, A., 2005. Modeling the interactions of small GTPases. Master's thesis, University of British Columbia, Vancouver.

Kimura, K., Ito, M., Amano, M., Chihara, K., Fukata, Y., Nakafuku, M., Yamamori, B., Feng, J., Nakano, T., Okawa, K., Iwamatsu, A., Kaibuchi, K., 1996. Regulation of myosin phosphatase by Rho and Rho-associated kinase (Rho-kinase). Science 273, 245-248.

Kraynov, V.S., Chamberlain, C., Bokoch, G.M., Schwartz, M.A., Slabaugh, S., Hahn, K.M., 2000. Localized Rac activation dynamics visualized in living cells. Science 290, 333-337.

Krishnan, J., Iglesias, P.A., 2004. A modeling framework describing the enzyme regulation of membrane lipids underlying gradient perception in Dictyostelium cells. J. Theor. Biol. 229, 85-99.

Kurokawa, K., Itoh, R.E., Yoshizaki, H., Nakamura, Y.O.T., Matsuda, M., 2004. Coactivation of Rac1 and $\mathrm{Cdc} 42$ at lamellipodia and membrane ruffles induced by epidermal growth factor. Mol. Biol. Cell 15, $1003-1010$.

Levchenko, A., Iglesias, P.A., 2002. Models of eukaryotic gradient sensing: application to chemotaxis of amoebae and neutrophils. Biophys. J. 82, 50-63.

Li, Z., Aizenman, C.D., Cline, H.T., 2002. Regulation of Rho GTPases by crosstalk and neuronal activity in vivo. Neuron $33,741-750$.

Li, Z., Dong, X., Wang, Z., Liu, W., Deng, N., Ding, Y., Tang, L., Hla, T., Zeng, R., Li, L., Wu, D., 2005. Regulation of PTEN by Rho small GTPases. Nat. Cell Biol. 7, 399-404.

Ma, L., Janetopoulos, C., Yang, L., Devreotes, P.N., Iglesias, P.A., 2004. Two complementary, local excitation, global inhibition mechanisms acting in parallel can explain the chemoattractant-induced regulation of PI(3,4,5) $\mathrm{P}_{3}$ response in Dictyostelium cells. Biophys. J. 87, 3764-3774.

Mackay, D.J.G., Hall, A., 1998. Rho GTPases. J. Biol. Chem. 273, 20685-20688. 
Marée, A.F.M., Jilkine, A., Dawes, A., Grieneisen, V.A., Edelstein-Keshet, L., 2006. Polarization and movement of keratocytes: a multiscale modeling approach. Bull. Math. Biol. 68, 1169-1211.

Matozaki, T., Nakanishi, H., Takai, Y., 2000. Small G-protein networks: their crosstalk and signal cascades. Cell. Signal. 12, 515-524.

Meili, R., Firtel, R.A., 2003. Two poles and a compass. Cell 114, 153-156.

Meinhardt, H., 1999. Orientation of chemotactic cells and growth cones: models and mechanisms. J. Cell Sci. 112, 2867-2874.

Michaelson, D., Silletti, J., Murphy, G., D’Eustachio, P., Rush, M., Philips, M.R., 2001. Differential localization of Rho GTPases in live cells: regulation by hypervariable regions and RhoGDI binding. J. Cell Biol. 152, 111-126.

Miki, H., Takenawa, T., 2003. Regulation of actin dynamics by WASP family proteins. J. Biochem. 134, 309-313.

Mitchison, T.J., Cramer, L.P., 1996. Actin-based cell motility and cell locomotion. Cell 84, 371-379.

Murray, J.D., 2002. Mathematical Biology I: An Introduction, 3rd edn. Springer, New York.

Nalbant, P., Hodgson, L., Kraynov, V., Toutchkine, A., Hahn, K.M., 2004. Activation of endogenous Cdc42 visualized in living cells. Science $305,1615-1619$.

Narang, A., 2006. Spontaneous polarization in eukaryotic gradient sensing: a mathematical model based on mutual inhibition of frontness and backness pathways. J. Theor. Biol. 240, 538-553.

Nobes, C.D., Hall, A., 1995. Rho, Rac, and Cdc42 GTPases regulate the assembly of multimolecular focal complexes associated with actin stress fibers, lamellipodia, and filopodia. Cell 81, 53-62.

Olofsson, B., 1999. Rho guanine dissociation inhibitors: pivotal molecules in cellular signalling. Cell. Signal. 11, 545-554.

Parent, C.A., Devreotes, P.N., 1999. A cell's sense of direction. Science 284, 765-770.

Pertz, O., Hodgson, L., Klemke, R.L., Hahn, K.M., 2006. Spatiotemporal dynamics of RhoA activity in migrating cells. Nature 440, 1069-1072.

Pestonjamasp, K.N., Forster, C., Sun, C., Gardiner, E.M., Bohl, B., Weiner, O., Bokoch, G.M., Glogauer, M., 2006. Rac1 links leading edge and uropod events through Rho and myosin activation during chemotaxis. Blood 108, 2814-2820.

Postma, M., Van Haastert, P.J.M., 2001. A diffusion-translocation model for gradient sensing by chemotactic cells. Biophys. J. 81, 1314-1323.

Postma, M., Bosgraaf, L., Loovers, H.M., Van Haastert, P.J.M., 2004. Chemotaxis: signalling modules join hands at front and tail. EMBO Rep. 5, 35-40.

Raftopoulou, M., Hall, A., 2004. Cell migration: Rho GTPases lead the way. Dev. Biol. 265, $23-32$.

Ridley, A.J., 2001a. Rho family proteins: coordinating cell responses. Trends Cell Biol. 11, 471-477.

Ridley, A.J., 2001b. Rho GTPases and cell migration. J. Cell Sci. 114, 2713-2722.

Ridley, A.J., Paterson, H.F., Johnston, C.L., Diekmann, D., Hall, A., 1992. The small GTP-binding protein Rac regulates growth factor-induced membrane ruffling. Cell 70, 401-410.

Rohatgi, R., Ma, L., Miki, H., Lopez, M., Kirchhausen, T., Takenawa, T., Kirschner, M.W., 1999. The interaction between N-WASP and the Arp2/3 complex links Cdc42-dependent signals to actin assembly. Cell 97, 221-231.

Sako, Y., Hibino, K., Miyauchi, T., Miyamoto, Y., Ueda, M., Yanagida, T., 2000. Single-molecule imaging of signaling molecules in living cells. Single Mol. 1, 159-163.

Sakumura, Y., Tsukada, Y., Yamamoto, N., Ishii, S., 2005. A molecular model for axon guidance based on cross talk between rho GTPases. Biophys. J. 89, 812-822.

Sander, E.E., Ten Klooster, J.-P., Van Delft, S., Van Der Kammen, R.A., Collard, J.G., 1999. Rac downregulates Rho activity: reciprocal balance between both GTPases determines cellular morphology and migratory behavior. J. Cell Biol. 147, 1009-1022.

Srinivasan, S., Wang, F., Glavas, S., Ott, A., Hofmann, F., Aktories, K., Kalman, D., Bourne, H.R., 2003. Rac and Cdc42 play distinct roles in regulating $\mathrm{PI}(3,4,5) \mathrm{P}_{3}$ and polarity during neutrophil chemotaxis. J. Cell Biol. 160, 375-385.

Stanyon, C.A., Bernard, O., 1999. LIM-kinase1. Int. J. Biochem. Cell Biol. 31, 389-394.

Subramanian, K.K., Narang, A., 2004. A mechanistic model for eukaryotic gradient sensing: spontaneous and induced phosphoinositide polarization. J. Theor. Biol. 231, 49-67.

Sun, C.X., Downey, G.P., Zhu, F., Koh, A.L.Y., Thang, H., Glogauer, M., 2004. Rac1 is the small GTPase responsible for regulating the neutrophil chemotaxis compass. Blood 104, 3758-3765.

Takai, Y., Sasaki, T., Matozaki, T., 2001. Small GTP-binding proteins. Physiol. Rev. 81, 153-208.

Thomas, R., 1981. On the relation between the logical structure of systems and their ability to generate multiple steady states or sustained oscillations. In: J. della Dora, J. Demongeot, B. Lacolle (Eds.), 
Numerical methods in the study of critical phenomena. Springer Series in Synergetics, vol. 9, pp. 180193. Springer, Berlin.

Thomas, R., Kaufman, M., 2001. Multistationarity, the basis of cell differentiation and memory. I. Structural conditions of multistationarity and other nontrivial behavior. Chaos 11, 170-179.

Tolias, K.F., Hartwig, J.H., Ishihara, H., Shibasaki, Y., Cantley, L.C., Carpenter, C.L., 2000. Type I $\alpha$ phosphatidylinositol-4-phosphate 5-kinase mediates Rac-dependent actin assembly. Curr. Biol. 10, 153-156.

Turing, A.M., 1952. The chemical basis of morphogenesis. Phil. Trans. Roy. Soc. Lond. B 237, 37-72.

Van Keymeulen, A., Wong, K., Knight, Z.A., Govaerts, C., Hahn, K.M., Shokat, K.M., Bourne, H.R., 2006. To stabilize neutrophil polarity, PIP3 and Cdc42 augment RhoA activity at the back as well as signals at the front. J. Cell Biol. 174, 437-445.

Van Leeuwen, F.N., Kain, H.E.T., Van Der Kammen, R.A., Michiels, F., Kranenburg, O.W., Collard, J.G., 1997. The guanine nucleotide exchange factor Tiam1 affects neuronal morphology; opposing roles for the small GTPases Rac and Rho. J. Cell Biol. 139, 797-807.

Verkhovsky, A.B., Svitkina, T.M., Borisy, G.G., 1999. Self-polarization and directional motility of cytoplasm. Curr. Biol. 9, 11-20.

Vidali, L., Chen, F., Cicchetti, G., Ohta, Y., Kwiatkowski, D.J., 2006. Rac1-null mouse embryonic fibroblasts are motile and respond to platelet-derived growth factor. Mol. Biol. Cell 17, 2377-2390.

Wedlich-Soldner, R., Altschuler, S., Wu, L., Li, R., 2003. Spontaneous cell polarization through actomyosin-based delivery of the Cdc42 GTPase. Science 299, 1231-1235.

Weiner, O.D., Neilsen, P.O., Prestwich, G.D., Kirschner, M.W., Cantley, L.C., Bourne, H.R., 2002. A PtdInsP $_{3}$ - and Rho GTPase-mediated positive feedback loop regulates neutrophil polarity. Nat. Cell Biol. 4, 509-513.

Wheeler, A.P., Wells, C.M., Smith, S.D., Vega, F.M., Henderson, R.B., Tybulewicz, V.L., Ridley, A.J., 2006. Rac1 and Rac2 regulate macrophage morphology but are not essential for migration. J. Cell Sci. 119, 2749-2757.

Wong, K., Pertz, O., Hahn, K., Bourne, H., 2006. Neutrophil polarization: spatiotemporal dynamics of RhoA activity support a self-organizing mechanism. Proc. Natl. Acad. Sci. U.S.A. 103, 3639-3644.

Xu, J., Wang, F., Van Keymeulen, A., Herzmark, P., Straight, A., Kelly, K., Takuwa, Y., Sugimoto, N., Mitchison, T., Bourne, H.R., 2003. Divergent signals and cytoskeletal assemblies regulate selforganizing polarity in neutrophils. Cell 114, 201-214.

Zerial, M., Huber, L.A. (Eds.) 1995. Guidebook to the Small GTPases. Oxford University Press, Oxford.

Zhang, B., Zheng, Y., 1998. Regulation of RhoA GTP hydrolysis by the GTPase-activating proteins p190, p50RhoGAP, Bcr, and 3BP-1. Biochemistry 37, 5249-5257.

Zheng, Y., 2001. Dbl family guanine nucleotide exchange factors. Trends Biochem. Sci. 26, 724-732.

Zondag, G.C.M., Evers, E.E., Ten Klooster, J.-P., Janssen, L., Van Der Kammen, R.A., Collard, J.G., 2000. Oncogenic Ras downregulates Rac activity, which leads to increased Rho activity and epithelialmesenchymal transition. J. Cell Biol. 149, 775-782. 